\title{
Vortex shedding in a two-dimensional diffuser: theory and simulation of separation control by periodic mass injection
}

\author{
By TAKAO SUZUKI ${ }^{1}$, TIM COLONIUS ${ }^{1}$, \\ AND SERGIO PIROZZOLI ${ }^{2}$ \\ ${ }^{1}$ Division of Engineering and Applied Science, California Institute of Technology, \\ Pasadena, CA 91125, USA \\ ${ }^{2}$ Department of Mechanics and Aeronautics, University of Rome 'La Sapienza', Rome, 00184, Italy
}

(Received 7 April 2003 and in revised form 4 August 2004)

We develop a reduced-order model for large-scale unsteadiness (vortex shedding) in a two-dimensional diffuser and use the model to show how periodic mass injection near the separation point reduces stagnation pressure loss. The model estimates the characteristic frequency of vortex shedding and stagnation pressure loss by accounting for the accumulated circulation due to the vorticity flux into the separated region. The stagnation pressure loss consists of two parts: a steady part associated with the time-averaged static pressure distribution on the wall, and an unsteady part caused by vortex shedding. To validate the model, we perform numerical simulations of compressible unsteady laminar diffuser flows in two dimensions. The model and simulation show good agreement as we vary the Mach number and the area ratio of the diffuser. To investigate the effects of periodic mass injection near the separation point, we also perform simulations over a range of the injection frequencies. Periodic mass injection causes vortices to be pinched off with a smaller size as observed in experiments. Consequently, their convective velocity is increased, absorption of circulation from the wall is enhanced, and the reattached point is shifted upstream. Thus, in accordance with the model, the stagnation pressure loss, particularly the unsteady part, is substantially reduced even though the separation point is nearly unchanged. This study helps explain experimental results of separation control using unsteady mass injection in diffusers and on airfoils.

\section{Introduction}

To achieve high performance in aircraft propulsion systems, it is crucial to minimize the stagnation pressure loss across an inlet diffuser. In many situations the length of the diffuser is restricted (for example, by low-observability constraints), and turning as well as rapid area changes are required (cf. Hamstra et al. 2000; MacMartin et al. 2001). As a result, large adverse pressure gradients between the throat and compressor cause flow separation. Previous experimental studies (e.g. Reneau, Johnston \& Kline 1967) have been used to categorize stall regimes for a planar diffuser geometry. In the so-called transitory stall regime, circulation is irregularly built up and washed out; thus, the flow pattern has similar features to vortex shedding from a bluff body, but sizes of the vortices are not uniform (see figure 1). Some experiments (e.g. Salmon, Bogar \& Sajben 1983) have visualized such a flow pattern. This 


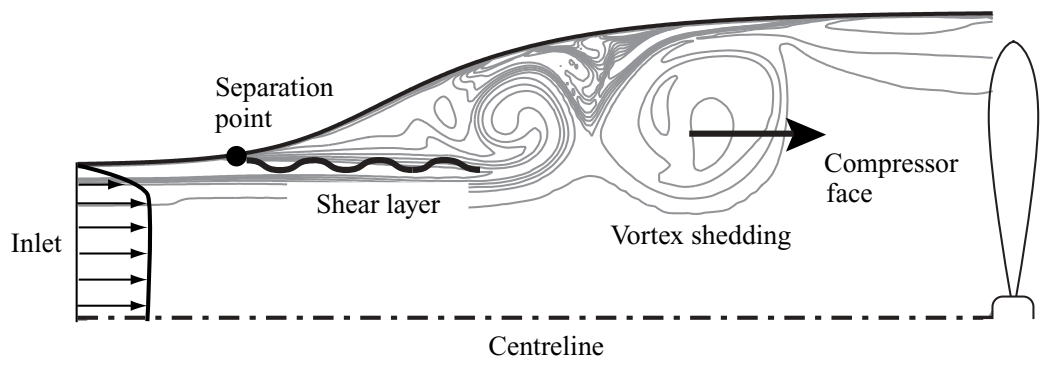

FIGURE 1. Schematic of vortex shedding in a two-dimensional diffuser.

phenomenon substantially reduces stagnation pressure and distorts the flow delivered to the compressor.

In the past, a number of studies have demonstrated that the pressure recovery can be improved if the generation of large-scale vortical disturbances is disrupted. Early experimental studies have accomplished this by using splitter vanes (Rao 1971), steady mass injection (Nicoll \& Ramaprian 1970; Back \& Cuffel 1982; Nishi, Yoshida \& Morimitsu 1998), a star tail-pipe (Welsh 1976), and moving walls (Tennant 1973). More recently, periodic mass injection (e.g. synthetic jets) near the separation point has been shown to enhance pressure recovery more effectively than previous control techniques (cf. Glezer \& Amitay 2002). This technique was introduced for airfoil separation (Seifert, Darabi \& Wygnanski 1996) and has also been applied to internal flows, such as diffusers (Amitay, Pitt \& Glezer 2002; Narayanan \& Banaszuk 2003).

The objective of this study is to investigate the large-scale vortex shedding intrinsic to transitory stall, and to study the mechanisms whereby periodic mass injection enhances performance. We develop a reduced-order model to predict the characteristic frequency of vortex shedding by accounting for the accumulated circulation due to the vorticity flux into the separated region. At low Mach numbers, the model also estimates the stagnation pressure loss, which consists of steady and unsteady parts: the former accounts for the time-averaged static pressure distribution in the separation region, and the latter for the vortex shedding. To verify the theoretical analyses, we perform direct numerical simulations (DNS) of two-dimensional unsteady laminar diffuser flows at various inflow Mach numbers $\left(M_{1}=0.2\right.$ to 0.8$)$ and area ratios $\left(h_{2} / h_{1}=1.4\right.$ to 2.6$)$.

The numerical simulations are also used to investigate the effects of periodic mass injection. This is modelled in a simplified way by locally forcing the flow inside the boundary layer upstream of the separation point. Over a range of frequencies, it is observed that periodic forcing causes frequency locking to occur (i.e. vortex shedding becomes periodic) with substantial reductions in stagnation pressure loss. The optimal forcing frequency is found to be about twice the natural vortex-shedding frequency, and the stagnation pressure loss can be approximately halved relative to the natural case (with an estimated forcing magnitude of $C_{\mu} \equiv\left(\overline{\rho u^{2}} h\right)_{\text {actuator }} /\left(\rho u^{2} h\right)_{\text {inflow }}=0.5 \%$ ).

Furthermore, we apply the model of diffuser flows to study the mechanisms of separation control. In fact, the model developed for transitory stall even more closely represents flows controlled by periodic mass injection. By estimating parameters in the model from the numerical simulation, we evaluate the contributions from the steady and unsteady parts of stagnation pressure loss as a function of the forcing frequency. In turn, this shows the mechanisms by which stagnation pressure loss is reduced when the separated boundary layer is pinched off into smaller vortices, and 


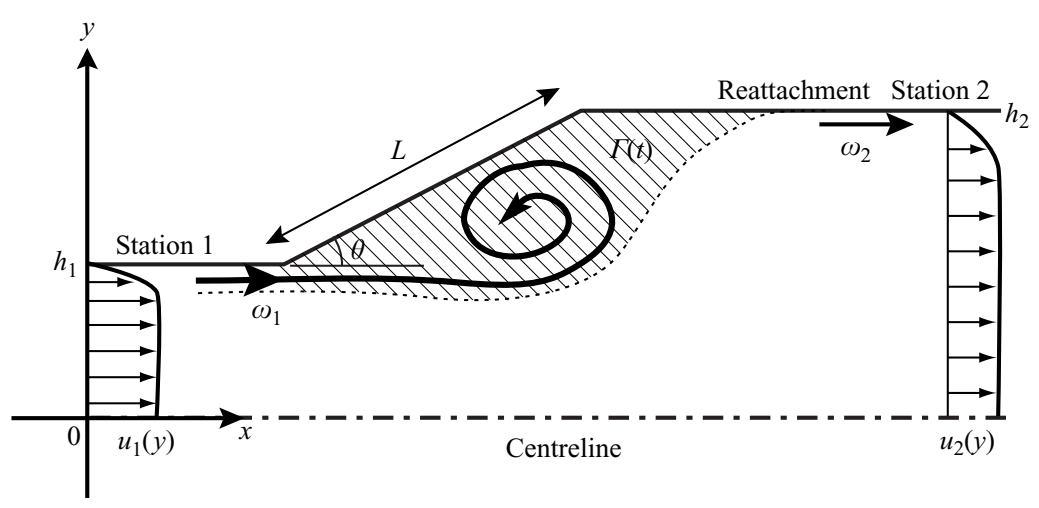

FIGURE 2. Simple model for a two-dimensional diffuser.

may explain the frequency dependence of pressure recovery observed in experiments. Although we carry out the analyses and simulations in two dimensions, we believe that the implications are applicable to three-dimensional flows. Especially when mass is injected uniformly across the spanwise direction, vortical structures downstream of the separation point appear to become two-dimensional as observed in experiments (cf. Amitay et al. 2002; Narayanan \& Banaszuk 2003).

The remainder of the paper is organized as follows. In the next section, the theoretical model is introduced. In $\S 3$, the methodology of the numerical simulation is briefly presented. The numerical results are compared with the model in $\S 4$, and the implications for separation control with periodic mass injection are discussed. Conclusions and limitations of the present analysis are given in $\S 5$.

\section{Model of a two-dimensional diffuser}

\subsection{Mechanism of vortex shedding}

To estimate the characteristic frequency of vortex shedding, we develop a reducedorder model for a two-dimensional diffuser. To simplify the discussion, we explain an incompressible model first and consider a compressible model in the following section.

\subsubsection{Incompressible flow model}

We consider a simple two-dimensional symmetric diffuser geometry and assume incompressible and inviscid flows. Taking a control volume and coordinate system as shown in figure 2, we estimate the accumulated circulation per unit time due to the vorticity flux through the inlet and exit. Of course, some vorticity flux is generated or absorbed at the diffuser wall owing to the no-slip boundary condition, but this mechanism is discussed in $\S 2.3$.

We specify a transversely sheared velocity profile at the inflow by $u_{1}(y)$ and assume the inflow pressure to be uniform (denoted by $p_{1}$ ). Furthermore, we assume that in the diffuser the separated boundary layer reattaches before it reaches the exit (station 2 in figure 2). The exit velocity profile is similarly specified as $u_{2}(y)$. Consequently, the net circulation accumulated in the control volume, per unit time, may be calculated as

$$
\frac{\mathrm{d} \Gamma}{\mathrm{d} t} \equiv \int_{0}^{h_{1}} \omega_{1}(y) u_{1}(y) \mathrm{d} y-\int_{0}^{h_{2}} \omega_{2}(y) u_{2}(y) \mathrm{d} y=\frac{u_{1}^{2}(0)-u_{2}^{2}(0)}{2},
$$


where the argument '( 0 )' emphasizes that the quantity is evaluated at the centreline. It is important to notice that the net vorticity flux is independent of the velocity profiles of the boundary layer.

During one period of vortex shedding, we assume that the accumulated circulation forms a single vortex in the hatched region in figure 2. In a viscous flow, some vorticity flux is absorbed from the wall, and this fraction is expressed by $\lambda$ (which will be formulated in (2.28)). We also assume that the diameter of the vortex can be scaled as $\left(h_{2}-h_{1}\right)$. Using Stokes' theorem, the velocity at the outer radius of this vortex can now be estimated as

$$
u_{v o r} \approx \frac{(1-\lambda)\left(u_{1}^{2}(0)-u_{2}^{2}(0)\right) T_{\text {shed }}}{2 \pi\left(h_{2}-h_{1}\right)},
$$

where $T_{\text {shed }}$ denotes the time period of vortex shedding. When this velocity exceeds a threshold velocity scaled by the free-stream velocity (denoted by $\alpha u_{1}(0)$ ), we hypothesize that the vortex is pinched off. Thus, the time period of vortex shedding in the incompressible case can be estimated as

$$
T_{\text {shed }}^{M \rightarrow 0} \approx \frac{\alpha}{1-\lambda} \frac{2 \pi h_{1}\left(h_{2} / h_{1}\right)^{2}}{u_{1}(0)\left(1+h_{2} / h_{1}\right)} .
$$

This equation shows that the shedding period becomes longer as the area ratio increases.

We note in passing that several experimental studies (e.g. Smith \& Kline 1974; Smith 1978; Kwong \& Dowling 1994) suggested that the proportionality of the stall period in a two-dimensional diffuser can be expressed as

$$
T_{\text {stall }} \sim \frac{L \sin 2 \theta}{u_{1}},
$$

where $L$ and $\theta$ are defined in figure 2 . If the area ratio is sufficiently large (i.e. $\left.h_{2} / h_{1} \gg 1\right)$ and the slope of the diffuser is small, namely, $\sin 2 \theta \sim 2 \sin \theta$, (2.3) yields the same scaling as (2.4). Note that some of these previous papers studied much longer time periods associated with the coupling between the diffuser and the dynamics of the whole system (i.e. resonance). Bogar, Sajben \& Kroutil (1983) discussed the distinction of such time scales. We focus only on the time period of vortex shedding in this study, which is thought to be more relevant to separation flow control.

\subsubsection{Compressible flow model}

We extend the discussion on vortex shedding above for compressible flows. To estimate the accumulated circulation, it is appropriate to calculate the flux of $\omega / \rho$ (cf. $\S 1.5$ of Saffman 1992), since Kelvin's circulation theorem ensures that this quantity is conserved in an inviscid barotropic fluid. Here, we additionally specify the transversely sheared temperature profiles at the inflow and exit as $T_{1}(y)$ and $T_{2}(y)$, respectively; accordingly, the density is given from the ideal gas law as $\rho_{1}(y)$. Thus, the equation corresponding to (2.1) becomes

$$
\begin{aligned}
\frac{1}{\bar{\rho}} \frac{\mathrm{d} \Gamma}{\mathrm{d} t} & \equiv \int_{0}^{h_{1}} \frac{\omega_{1}(y)}{\rho_{1}(y)} u_{1}(y) \mathrm{d} y-\int_{0}^{h_{2}} \frac{\omega_{2}(y)}{\rho_{2}(y)} u_{2}(y) \mathrm{d} y \\
& =\frac{R}{p_{1}} \int_{0}^{u_{1}(0)} T_{1}\left(u_{1}\right) u_{1} \mathrm{~d} u_{1}-\frac{R}{p_{2}} \int_{0}^{u_{2}(0)} T_{2}\left(u_{2}\right) u_{2} \mathrm{~d} u_{2},
\end{aligned}
$$

where $\bar{\rho}$ on the left-hand side denotes a characteristic density scale (which will be specified in (2.8)), and $R$ is the gas constant. 
To evaluate the temperature profile, we use the Crocco-Busemann relation (cf. $\S X V$ of Schlichting 1960). Assuming that the Prandtl number is unity and the flow is parallel, the temperature profile above an iso-thermal wall may be calculated as a function of velocity:

$$
\frac{T(u)}{T_{\infty}}=\frac{T_{\text {wall }}}{T_{\infty}}+\left(1-\frac{T_{w a l l}}{T_{\infty}}+\frac{u_{\infty}^{2}}{2 c_{p} T_{\infty}}\right) \frac{u}{u_{\infty}}-\frac{u_{\infty}^{2}}{2 c_{p} T_{\infty}}\left(\frac{u}{u_{\infty}}\right)^{2},
$$

where $c_{p}$ represents the specific heat at constant pressure. In addition, the subscript $\infty$ denotes quantities just outside the boundary layer, which we now approximate by the corresponding centreline quantities (e.g. $\left.u_{\infty}=u(0)\right)$. $T_{\text {wall }}$ denotes the wall temperature, which is assumed constant everywhere. Substituting (2.6) into (2.5) yields

$$
\begin{aligned}
\frac{1}{\bar{\rho}} \frac{\mathrm{d} \Gamma}{\mathrm{d} t}=\frac{u_{1}^{2}(0)}{2 \rho_{1}(0)}\left[\frac{2}{3}+\frac{1}{3} \frac{T_{\text {wall }}}{T_{1}(0)}+\right. & \left.\frac{(\gamma-1) M_{1}^{2}(0)}{12}\right] \\
& -\frac{u_{2}^{2}(0)}{2 \rho_{2}(0)}\left[\frac{2}{3}+\frac{1}{3} \frac{T_{\text {wall }}}{T_{2}(0)}+\frac{(\gamma-1) M_{2}^{2}(0)}{12}\right] .
\end{aligned}
$$

Here, $M_{j} \equiv u_{j} / \sqrt{\gamma R T_{j}}(j=1$ or 2$)$ is the Mach number.

Following the same argument as in the preceding section and assuming the averaged density of the vortex, $\bar{\rho}$ in $(2.5)$, to be $\rho_{1}(0)$ (in fact, it is still lower owing to compressibility), the time period of vortex shedding for compressible flows can be estimated as

$$
T_{\text {shed }} \approx \frac{\alpha}{1-\lambda} \frac{2 \pi h_{1}\left(h_{2} / h_{1}-1\right)}{u_{1} F},
$$

where

$$
\begin{aligned}
F\left(M_{1}, \frac{h_{2}}{h_{1}}, \frac{u_{2}}{u_{1}}\right. & \left.\frac{T_{2}}{T_{1}}, \frac{T_{\text {wall }}}{T_{1}}\right) \equiv\left[\frac{1}{2}+\frac{1}{3} \frac{T_{\text {wall }}}{T_{1}}+\frac{1}{6}\left(1+\frac{\gamma-1}{2} M_{1}^{2}\right)\right] \\
& -\left(\frac{h_{2}}{h_{1}}\right)\left(\frac{u_{2}}{u_{1}}\right)^{3}\left[\frac{1}{2}+\frac{1}{3} \frac{T_{\text {wall }}}{T_{1}} \frac{T_{1}}{T_{2}}+\frac{1}{6}\left(1+\frac{\gamma-1}{2} M_{1}^{2}\right) \frac{T_{1}}{T_{2}}\right] .
\end{aligned}
$$

In these equations, it is assumed that the boundary layer is sufficiently thin that the centreline quantities, denoted by $(0)$ in the discussion above, are now given by a quasi-one-dimensional analysis:

$$
\begin{aligned}
\rho_{1} u_{1} h_{1} & =\rho_{2} u_{2} h_{2}, \\
c_{p} T_{1}+\frac{1}{2} u_{1}^{2} & =c_{p} T_{2}+\frac{1}{2} u_{2}^{2} .
\end{aligned}
$$

Thus, we treat quantities with a subscript as a cross-sectional average in (2.8) and (2.9) here. More precisely, we must take time averages of these quantities over a period of vortex shedding as well. Using (2.10) and (2.11), we can specify $u_{2} / u_{1}$ and $T_{2} / T_{1}$ in (2.9) as functions of $h_{2} / h_{1}$ and $p_{2} / p_{1}$.

Figure 3 plots the shedding time period as a function of the area ratio at various inflow Mach numbers. Here, we calculate $p_{2} / p_{1}$ based on the ideal expansion and assume $T_{\text {wall }}=T_{1}$ and no absorption of circulation from the wall $(\lambda=0)$. The normalized shedding period decreases as the inflow Mach number increases. This compressibility effect is appreciable when $h_{2} / h_{1}$ is small; however, it becomes small at lower Mach numbers or in higher area ratios. 


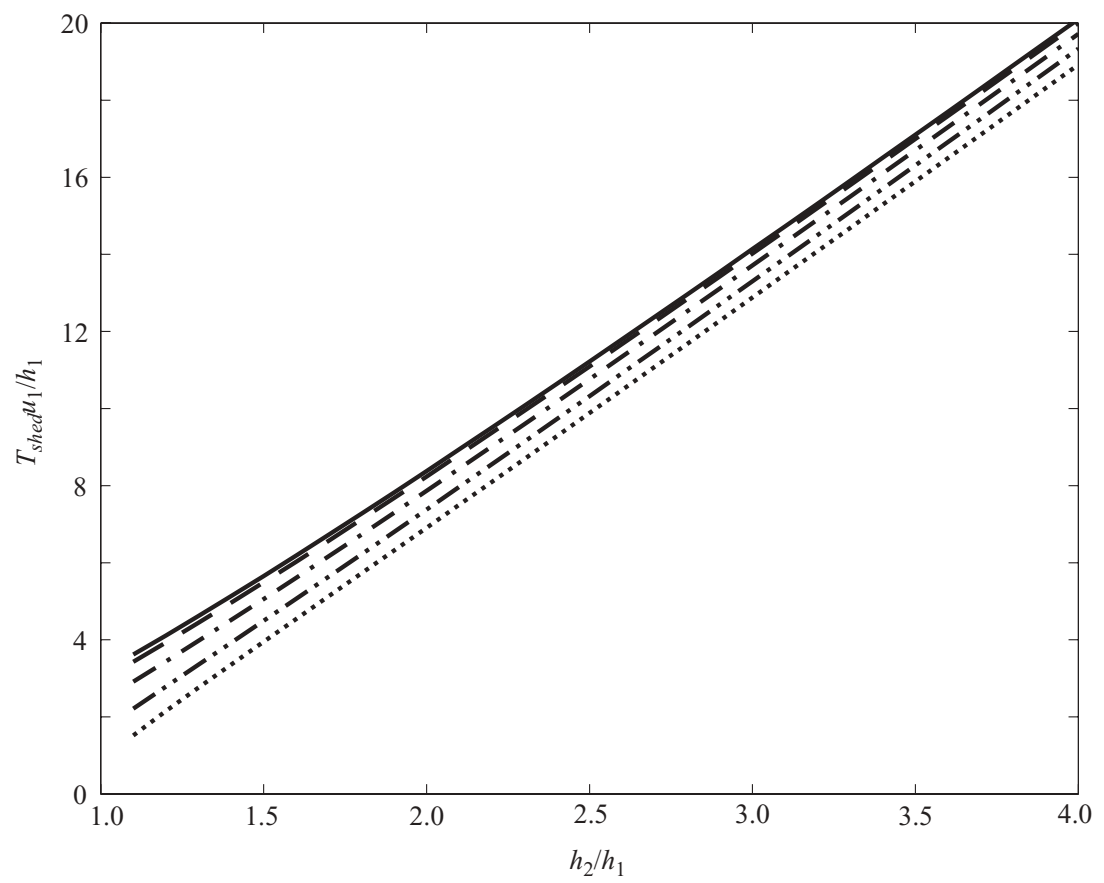

FIGURE 3. The vortex-shedding time period as a function of the area ratio for various inflow Mach numbers: - , incompressible limit;,$--- M_{1}=0.2 ;-\cdot-, M_{1}=0.4 ;-\cdot--, M_{1}=0.6$; $\cdots, M_{1}=0.8 . \alpha=1$ and $\lambda=0$ are assumed.

\subsection{Stagnation pressure loss in a diffuser}

In this section, we evaluate stagnation pressure loss due to flow separation and largescale flow unsteadiness in the incompressible limit. We start with Crocco's equation:

$$
\frac{\partial u_{i}}{\partial t}+\frac{\partial H}{\partial x_{i}}-T \frac{\partial s}{\partial x_{i}}=\epsilon_{i j k} u_{j} \omega_{k}+\frac{1}{\rho} \frac{\partial \tau_{i j}}{\partial x_{j}},
$$

where $H$ is total enthalpy ( $\left.\equiv c_{p} T+u_{k}^{2} / 2\right)$, $s$ is entropy, and $\tau_{i j}$ is the viscous stress tensor. We define stagnation temperature to be $T_{t} \equiv H / c_{p}$, and accordingly, stagnation pressure and density to be $p_{t} \equiv\left(T_{t} / T\right)^{\gamma /(\gamma-1)} p$ and $\rho_{t} \equiv\left(T_{t} / T\right)^{1 /(\gamma-1)} \rho$, respectively. As a result, (2.12) can be rewritten as

$$
\frac{\partial p_{t}}{\partial x_{i}}=\rho_{t} \epsilon_{i j k} u_{j} \omega_{k}-\rho_{t} \frac{\partial u_{i}}{\partial t}+\left(T-T_{t}\right) \rho_{t} \frac{\partial s}{\partial x_{i}}+\frac{\rho_{t}}{\rho} \frac{\partial \tau_{i j}}{\partial x_{j}} .
$$

Note that entropy is the same between static and stagnation quantities by definition. Now, we evaluate the order of each term in (2.13). We assume that the compressibility is weak and expand both static and stagnation thermodynamic quantities for small $M^{2}$ (cf. Chu \& Kovásznay 1958). For example, $\left(p-p_{\infty}\right) /\left(\rho_{\infty} u_{1}^{2} / 2\right) \equiv \tilde{p}_{M^{0}}+M^{2} \tilde{p}_{M^{2}}+O\left(M^{4}\right)$, etc. In addition, we assume that the time scale of the fluid motion is proportional to '(length scale) $/\left(\right.$ velocity scale)', namely $(\partial / \partial t) /(\partial / \partial x) \sim u_{1}$. The first two terms on the right-hand side of (2.13) then yield $O(1)$. The third term can be estimated as $O\left(M^{2} / R e\right)$ referring to the entropy change along the material line (if the flow is not initially homoentropic, its order is $O\left(M^{2}\right)$ ). Likewise, the last term can be evaluated as $O(1 / R e)$. Thus, assuming $R e \gg 1$ and neglecting $O\left(M^{2}\right)$ and higher-order terms, 


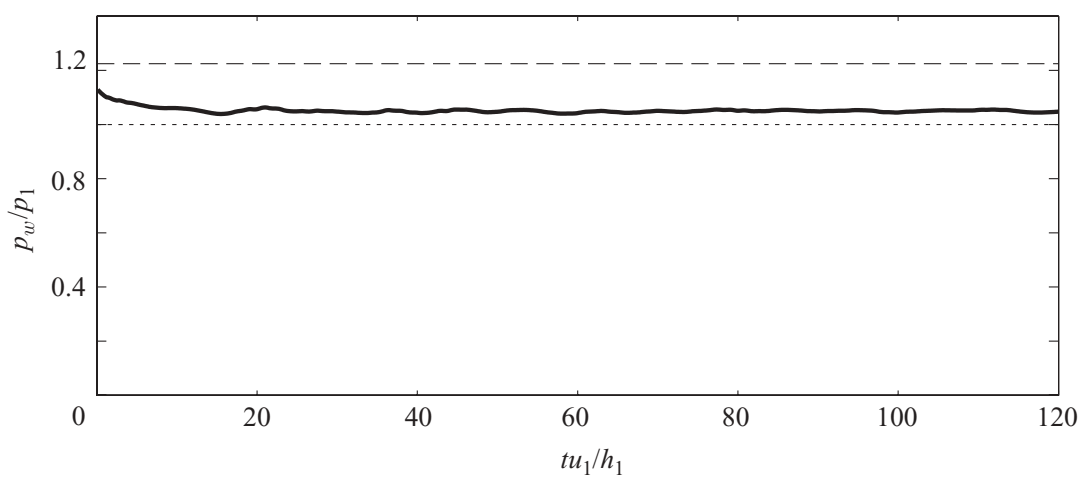

FiguRE 4. Projected pressure on the upper wall in the $x$-direction (denoted by $\longrightarrow$ ). Static pressure on the wall was integrated over $x / h_{1} \in[-2,3]$ on the upper wall in simulation (case A, see figure 11 for the flow field). The actual integrated area in simulation was $\left(h_{2}-h_{1}\right) / h_{1}=0.889$, and it is taken into account. $p_{2} / p_{1}$ is denoted by --- .

(2.13) can be approximated as

$$
\frac{\partial p_{t}}{\partial x_{i}} \approx \rho_{t} \epsilon_{i j k} u_{j} \omega_{k}-\rho_{t} \frac{\partial u_{i}}{\partial t} .
$$

Next, we integrate (2.14) for $i=1$ (the $x$-direction) inside the control volume defined in figure 2. Using Green's theorem, the left-hand side yields the surface integrals. Integrating it over the cross-section, the second term on the right-hand side vanishes for a symmetric vortex, as discussed later. Alternatively, if we take a time average and expand this term as $\left.\left.\overline{\left(\rho_{\infty}-\rho_{t}\right)(\partial u / \partial t}\right)-\overline{\rho_{\infty}(\partial u / \partial t}\right)$, it is evaluated as $O\left(M^{2}\right)$. Consequently, the stagnation pressure loss averaged over the cross-section can be approximated as

$$
p_{t 1}-p_{t 2} \approx\left(p_{t 1}-p_{w}\right)\left(1-\frac{h_{1}}{h_{2}}\right)-\frac{\iint \rho_{t} v \omega \mathrm{d} x \mathrm{~d} y}{h_{2}},
$$

where the averaged pressure projected on the wall is defined as $p_{w} \equiv \int_{h_{1}}^{h_{2}} p \mathrm{~d} y /\left(h_{2}-h_{1}\right)$. As mentioned in the previous section, quantities with a subscript denote the crosssectional average. This equation shows that stagnation pressure loss can be decreased by increasing either $p_{w}$ or $\iint \rho_{t} v \omega \mathrm{d} x \mathrm{~d} y$, and these two terms are analysed in the following.

\subsubsection{Steady part of stagnation pressure loss}

First, we consider the first term on the right-hand side of (2.15). In a rapidly diverging diffuser, the boundary layer is fully separated. Figure 4 demonstrates $p_{w} \approx p_{1}$, although pressure on the wall is slightly underestimated (approximately $4.9 \%$ ) because the free-stream pressure is actually greater than $p_{1}$ at the separation point. In fact, figure 5 shows that the error of $(2.15)$ on average is about $2.1 \%$ of the inlet stagnation pressure if we assume $p_{w}=p_{1}$. The slight underestimate of pressure on the wall causes an overestimate of the stagnation pressure loss, but the error is very small and stationary over time; hence, the neglected terms in (2.14) are considered small.

Next, the steady part of $\iint \rho_{t} v \omega \mathrm{d} x \mathrm{~d} y$ can also be readily evaluated in incompressible flows. Imposing the no-slip boundary conditions on the wall, this term can 


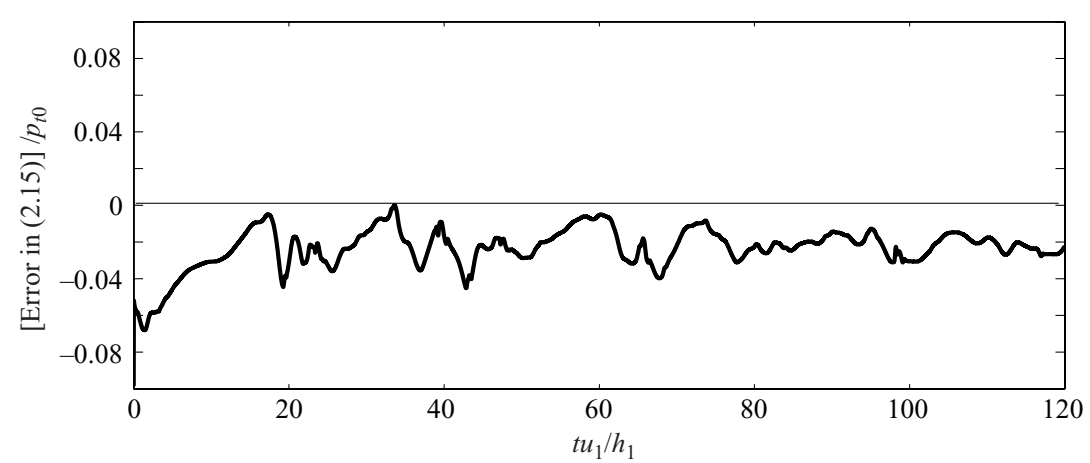

FIGURE 5. Error in the estimate of stagnation pressure loss. Referring to (2.15), (1.h.s. r.h.s. $) / p_{t 0}$ is plotted, where $p_{t 0} \equiv\left(1+((\gamma-1) / 2) M_{1}^{2}\right)^{\gamma /(\gamma-1)} p_{1}$. Correction of the projected area mentioned in figure 4 is taken into account.

be expanded as follows:

$$
\begin{aligned}
\iint \rho_{t} v \omega \mathrm{d} x \mathrm{~d} y & \approx \iint \rho_{\infty} v\left(\frac{\partial v}{\partial x}-\frac{\partial u}{\partial y}\right) \mathrm{d} x \mathrm{~d} y \\
& =\rho_{\infty} \iint\left(\frac{\partial\left(v^{2} / 2\right)}{\partial x}-\frac{\partial(u v)}{\partial y}+u \frac{\partial v}{\partial y}\right) \mathrm{d} x \mathrm{~d} y \\
& =-\rho_{\infty} \iint \frac{\partial\left(u^{2} / 2\right)}{\partial x} \mathrm{~d} x \mathrm{~d} y=\rho_{\infty} \frac{u_{1}^{2} h_{1}-u_{2}^{2} h_{2}}{2} .
\end{aligned}
$$

Here, continuity is used, and $v_{1}=v_{2}=0$ is assumed for the third equality. This relation is nothing but a momentum balance in the control volume (cf. $\S 5.15$ of Batchelor 1973). Thus, the steady part of the time-averaged stagnation pressure loss can be expressed as

$$
\begin{aligned}
\left(\overline{p_{t 1}-p_{t 2}}\right)_{\text {steady }}^{M \rightarrow 0} & \approx \frac{\left(p_{1}-\overline{p_{w}}\right)\left(h_{2} / h_{1}-1\right)}{h_{2} / h_{1}}+\frac{1}{2} \rho u_{1}^{2} \frac{\left(h_{2} / h_{1}-1\right)^{2}}{\left(h_{2} / h_{1}\right)^{2}} \\
& =\frac{1}{2} \rho u_{1}^{2} \frac{\left(h_{2} / h_{1}-1\right)^{2}}{\left(h_{2} / h_{1}\right)^{2}} \frac{\overline{p_{w}^{*}}-\overline{p_{w}}}{\overline{p_{w}^{*}}-p_{1}}
\end{aligned}
$$

where

$$
\overline{p_{w}^{*}} \equiv p_{1}+\frac{1}{2} \rho u_{1}^{2} \frac{h_{2} / h_{1}-1}{h_{2} / h_{1}}
$$

is the highest possible time-averaged pressure projected on the wall, assuming a steady, irrotational and ideal flow. Therefore, this is only achieved if the flow is assumed perfectly attached, in which case $\left(\overline{p_{t 1}-p_{t 2}}\right)_{\text {steady }}^{M \rightarrow 0}=0$.

\subsubsection{Unsteady part of stagnation pressure loss}

The unsteady part of the $\iint \rho_{t} v \omega \mathrm{d} x \mathrm{~d} y$ term is considered to be the contribution from vortex shedding. To analyse this effect, we use the Oseen vortex (Oseen 1912) as a model. The Oseen vortex is a solution to the incompressible Navier-Stokes equations in an otherwise quiescent flow. Again, we consider the leading terms of the incompressible limit. The azimuthal velocity of the Oseen vortex is given by

$$
u_{\theta}(t, x, y)=\frac{\Gamma}{2 \pi \sqrt{\left(x-x_{0}\right)^{2}+\left(y-y_{0}\right)^{2}}}\left(1-\exp \left[-\frac{\left(x-x_{0}\right)^{2}+\left(y-y_{0}\right)^{2}}{4 v t}\right]\right),
$$




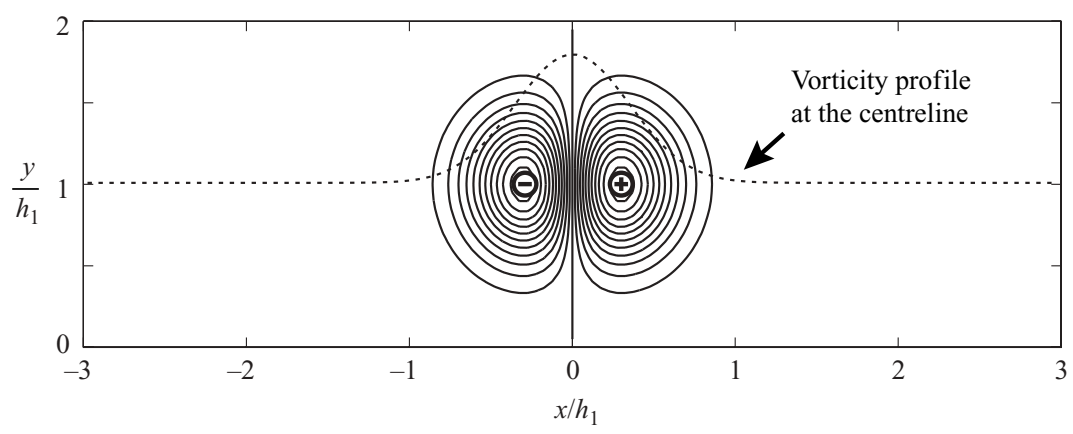

Figure 6. Contours of $\left(\rho_{t} v \omega\right) /\left(\rho u_{1}^{2} / 2\right)$ for the Oseen vortex are shown. Parameters are chosen according to case A shown in table $1\left(h_{2} / h_{1}=2\right)$, and $\alpha=1$ is assumed. The centre of the vortex is $\left(x_{0} / h_{1}, y_{0} / h_{1}\right)=(0,1)$. Contour level: minimum, -6.0 ; maximum, 6.0 ; and the interval of 0.5 .

where $\left(x_{0}, y_{0}\right)$ denotes the centre of the vortex, $\Gamma$ is the total circulation, and $v$ is the kinematic viscosity. Here, we assume that the time scale of dissipation is much slower than that of vortex convection; accordingly, we assume the radius of the vortex, $R_{v} \equiv \sqrt{4 v t}$, to be constant. Using this expression, we can derive

$$
\rho_{t} v \omega=\frac{\rho_{t} \Gamma^{2}}{2 \pi^{2} R_{v}^{2}} \frac{x-x_{0}}{r^{2}}\left(\exp \left[-\frac{r^{2}}{R_{v}^{2}}\right]-\exp \left[-\frac{2 r^{2}}{R_{v}^{2}}\right]\right),
$$

where $r^{2} \equiv\left(x-x_{0}\right)^{2}+\left(y-y_{0}\right)^{2}$. Assuming that the effect of blockage due to the wall is weak (which can be confirmed from a distribution of $\rho_{t} v \omega$ in figure 6), we integrate (2.20) in the vertical direction. After some algebra, we obtain

$$
\int_{-\infty}^{\infty} \rho_{t} v \omega \mathrm{d} y=\frac{\rho_{t} \Gamma^{2}}{2 \pi R_{v}^{2}}\left(\operatorname{erf}\left[\frac{\sqrt{2}\left(x-x_{0}\right)}{R_{v}}\right]-\operatorname{erf}\left[\frac{x-x_{0}}{R_{v}}\right]\right),
$$

where $\operatorname{erf}(z) \equiv(2 / \sqrt{\pi}) \int_{0}^{z} \exp \left(-t^{2}\right) \mathrm{d} t$. Notice that $(2.21)$ is positive downstream of the vortex centre $\left(x-x_{0}>0\right)$ and negative upstream of it $\left(x-x_{0}<0\right)$ (see figure 6). Therefore, when the vortex leaves the control volume, a stagnation pressure drop appears at the exit cross-section. According to the discussion in $\S 2.1$, we estimate the parameters as $\Gamma=\alpha 2 \pi R_{v} u_{1}$ and $R_{v} \approx\left(h_{2}-h_{1}\right) / 2$ and denote the convective velocity of the vortex as $u_{c}$. Thus, the stagnation pressure loss averaged over the exit cross-section for each vortex shedding is given as a function of time,

$$
\begin{aligned}
\left(\Delta p_{t 2}\right)_{\text {shed }}(t) & =\frac{1}{h_{2}} \int_{u_{c} t}^{\infty} \int_{-\infty}^{\infty} \rho_{t} v \omega \mathrm{d} y \mathrm{~d} x \\
& \approx \alpha^{2} \pi \rho_{\infty} u_{1}^{2} \frac{h_{2}-h_{1}}{h_{2}} \int_{\infty}^{u_{c} t / R_{v}}(\operatorname{erf}[\sqrt{2} \chi]-\operatorname{erf}[\chi]) \mathrm{d} \chi
\end{aligned}
$$

When the vortex is convected along $y=h_{2} / 2$, the convective velocity can be approximated as $u_{c}=u_{2}$. In reality, the vortex is pinched off near the upper wall and probably convected above $y=h_{2} / 2$ when $h_{2} / h_{1}<2$, and vice versa. Therefore, the convective velocity is reduced owing to the induced velocity of the image vortex. To be precise, the Oseen vortex is no longer a valid solution because the centre of the vortex is convected slower than the bulk flow. 


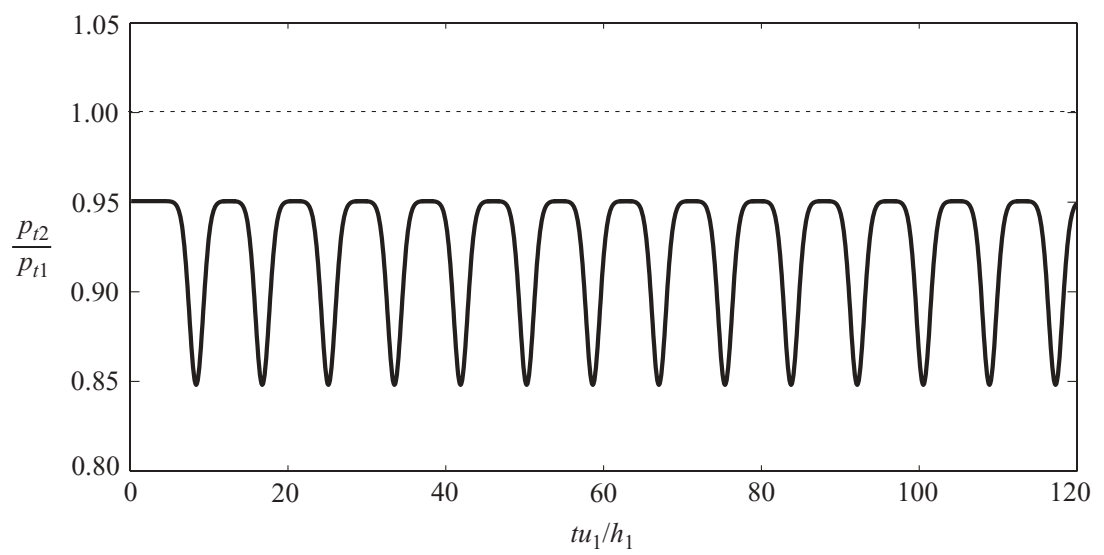

FiguRE 7. Stagnation pressure profile estimated based on the inviscid and incompressible limit. Parameters are chosen according to case $\mathrm{A}\left(M_{1}=0.6\right.$ and $\left.h_{2} / h_{1}=2\right)$, and $\alpha=1, \beta=1, \lambda=0$, $\overline{p_{w}}=p_{1}$ and $\kappa=0.33$ are assumed.

To estimate the induced velocity due to blockage, we assume an ideal fluid and consider a conformal mapping represented by $\zeta=\exp \left(\left(\pi / 2 h_{2}\right) z\right)$. This function maps an infinitely long channel onto the first quadrant; hence, two pairs of counter-rotating point vortices satisfy the non-penetration boundary condition in the $\zeta$-plane. According to Pierrehumbert's study (1980) on a pair of counter-rotating vortex 'patches', the convective velocities of distributed vortices can be approximated well by those of point vortices, even if the distance between them is of the same order as the vortex size. The rate of induced velocity for point vortices can be calculated as (cf. $§ 7.2$ of Saffman 1992)

$$
\kappa\left(\frac{h_{2}}{h_{1}}, \frac{h_{3}}{h_{1}}\right) \equiv-\frac{u_{\text {ind }}}{\alpha u_{2}} \approx-\frac{1}{4} \pi\left(\frac{h_{2}}{h_{1}}-1\right) \frac{1}{\tan \left(\pi\left(h_{3} / h_{1}\right)\left(h_{1} / h_{2}\right)\right)},
$$

where $h_{3}$ is the distance from the centreline to the vortex centre, and the convective velocity then becomes $(1-\alpha \kappa) u_{2}$. For example, when $h_{2} / h_{1}=2$ and $h_{3} / h_{1}=\left[\left(h_{1}+\right.\right.$ $\left.\left.h_{2}\right) / 2+h_{1}\right] /\left(2 h_{1}\right)=5 / 4, \kappa$ becomes as high as 0.33 compared to zero in a free space (for reference, if we simply calculate the infinite superposition of the Oseen vortices under the conditions of figure 6 , we obtain $\kappa=0.32$, which is sufficiently close to the point-vortex solution). Thus, the effect of blockage on the convective velocity is not negligible. On the other hand, the error of $\iint \rho_{t} v \omega \mathrm{d} x \mathrm{~d} y$ associated with blockage is less than $2 \%$.

Now, we assume that each vortex-shedding process is sufficiently isolated in time (which will be confirmed in figure 7). Subsequently, using (2.22) and knowing that the time period of vortex shedding is estimated by (2.3), the time-averaged stagnation pressure loss associated with vortex shedding can be estimated as

$$
\left(\overline{\Delta p_{t 2}}\right)_{\text {shed }}^{M \rightarrow 0}=\frac{\int_{-\infty}^{\infty} p_{t 2}(t) \mathrm{d} t}{T_{\text {shed }}^{(M \rightarrow 0)}} \approx-\frac{\alpha(1-\lambda)}{1-\alpha \kappa} \frac{\left(h_{2} / h_{1}+1\right)\left(h_{2} / h_{1}-1\right)^{2}}{8\left(h_{2} / h_{1}\right)^{2}} \frac{\rho u_{1}^{2}}{2} .
$$

This equation shows that the stagnation pressure loss due to vortex shedding is proportional to the dynamic pressure in the incompressible limit. 
Although we have derived (2.24) based on the Oseen vortex model, this dependence remains valid for any radially symmetric vortex, provided we define a coefficient

$$
\beta=\frac{8 \pi}{\Gamma^{2}} \int_{-\infty}^{\infty} \int_{\infty}^{\tau} \int_{-\infty}^{\infty} v \omega \mathrm{d} y \mathrm{~d} x \mathrm{~d} \tau,
$$

which relates the vorticity distribution to that of the Oseen vortex. In fact, $\beta$ is found to be close to unity in the numerical simulations (see table 3).

Accordingly, the total stagnation pressure loss is now expressed as

$$
\left(\overline{p_{t 1}-p_{t 2}}\right)_{\text {total }}^{M \rightarrow 0} \approx \frac{\rho u_{1}^{2}}{2} \frac{\left(h_{2} / h_{1}-1\right)^{2}}{\left(h_{2} / h_{1}\right)^{2}}\left[\frac{\overline{p_{w}^{*}}-\overline{p_{w}}}{\overline{p_{w}^{*}}-p_{1}}+\frac{\alpha \beta(1-\lambda)}{1-\alpha \kappa} \frac{h_{2} / h_{1}+1}{8}\right] .
$$

The first term in (2.26) corresponds to the steady loss, and the second term to the unsteady loss. As discussed later, (2.26) indicates that the contribution from vortex shedding becomes comparable to the steady part as the induced velocity from the image vortex reduces the convective velocity. Figure 7 shows a predicted pattern of the stagnation pressure profile for a fully separated case. The troughs correspond to the unsteady loss superposed on the steady part. The stagnation pressure profile drops when the centre of the vortex is passing through the exit cross-section (approximately $11 \%$ drop under the conditions corresponding to case A defined in table 1). These theoretical predictions are examined using numerical simulation in $\S 4.2$.

Although (2.26) is developed for natural vortex shedding, the formula can be applied to actively controlled cases by varying the parameters. For example, by periodically forcing the boundary layer, we can reduce the circulation of each vortex. This corresponds in the model to reducing $\alpha$, which, in turn, lessens stagnation pressure loss, as discussed in $\S 4.3$.

\subsubsection{Absorption of vorticity flux from the wall}

In a free space, the vorticity distribution is diffused, but the net circulation is conserved. Therefore, the dominant part of viscous effects should be caused by the vorticity flux on the wall. According to the discussion in $\S 2.1$, the accumulated circulation per unit time is equal to the net vorticity flux into the control volume. This now includes the vorticity flux through the wall, which can be expressed as (e.g. Koumoutsakos, Leonard \& Pépin 1994)

$$
\int_{\text {wall }} \text { (vorticity flux) } \mathrm{d} s=\int_{\text {wall }} \nu \frac{\partial \omega}{\partial n} \mathrm{~d} s=-\int_{\text {wall }} \frac{1}{\rho} \frac{\partial p}{\partial s} \mathrm{~d} s,
$$

where the positive sign is taken to be production. This expression is valid even in compressible flows when the dynamic viscosity $\mu(=\rho v)$ is constant everywhere. This term produces additional circulation in favourable pressure gradients, while in adverse ones it absorbs circulation or creates negative (counter-rotating) vorticity.

In incompressible flows, $(2.27)$ simply yields $\left(p_{1}-p_{2}\right)_{\text {wall }} / \rho$. Assuming $\left(p_{1}\right)_{\text {wall }}=p_{1}$ and taking a time average, the rate of absorption introduced in (2.2) can be expressed as

$$
\lambda \equiv-\frac{p_{1}-\left(\overline{p_{2}}\right)_{\text {wall }}}{\rho(\mathrm{d} \Gamma / \mathrm{d} t)}=\frac{\left(\overline{p_{2}}\right)_{\text {wall }}-p_{1}}{\frac{1}{2} \rho u_{1}^{2}} \frac{\left(h_{2} / h_{1}\right)^{2}}{\left(h_{2} / h_{1}\right)^{2}-1} .
$$

From the discussion in $\S 2.1 .1$ and Bernoulli's equation, the rate of accumulated circulation per unit time in the control volume is $\mathrm{d} \Gamma / \mathrm{d} t=\left(u_{1}^{2}-u_{2}^{2}\right) / 2=\left(p_{2}-p_{1}\right) / \rho$; therefore, if this amount is perfectly absorbed from the wall (i.e. $\lambda=1$ ), no vortex shedding occurs and the pressure on the wall coincides with the free-stream pressure, 

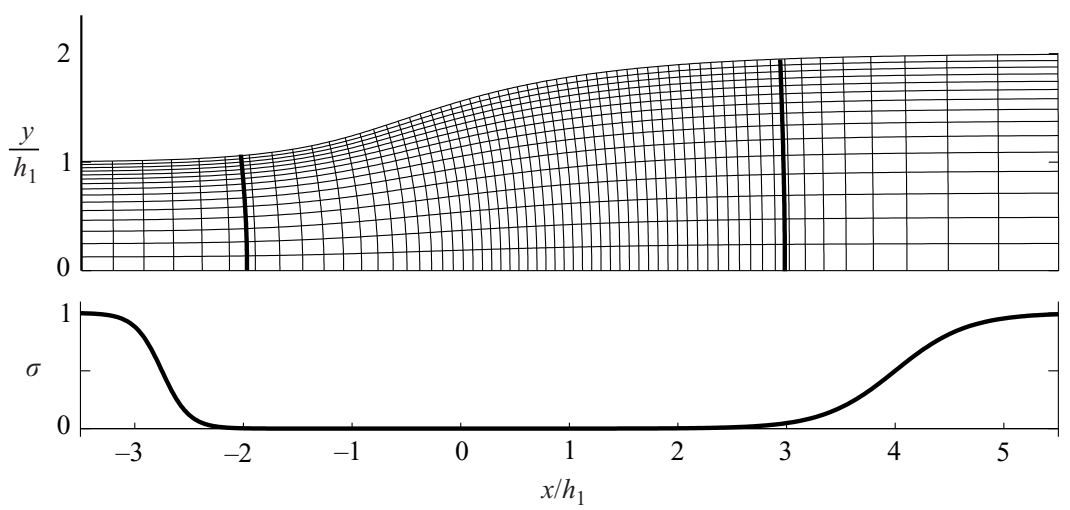

FIGURE 8. A typical computational grid on the top and the strength of the sponge term on the bottom. Every tenth grid point is shown for case A, and the thicker lines denote the sections where the stagnation pressure profiles were recorded. $\Delta x_{\min } / h_{1}=8.29 \times 10^{-3}, \Delta x_{\max } /$ $h_{1}=59.45 \times 10^{-3}, \Delta y_{\min } / h_{1}=5.57 \times 10^{-3}$ and $\Delta y_{\max } / h_{1}=25.15 \times 10^{-3}$.

i.e. $\overline{p_{w}}=\overline{p_{w}^{*}}$ in (2.17). However, when the flow is fully separated, vorticity is absorbed mainly near the reattachment point and the projection of recovered static pressure in the $x$-direction is reduced.

\section{Numerical procedures}

\subsection{Direct numerical simulation}

To simulate unsteady laminar diffuser flows, we performed direct numerical simulation (DNS) solving the compressible Navier-Stokes equations in two dimensions. The governing equations in curvilinear coordinates are cast in the strong conservation form (Vinokur 1974). The fourth-order Runge-Kutta scheme was used for time marching. For spatial derivatives, the sixth-order Padé scheme (Lele 1992) was used for the interior points with lower-order closures (third and fourth order) at the inflow, exit and wall boundaries.

A two-dimensional diffuser shape was generated by conformal mapping. The function

$$
z=\frac{\left(h_{2} / h_{1}\right)+1}{2} \bar{\zeta}+\frac{1}{c} \frac{\left(h_{2} / h_{1}\right)-1}{2} \log (\cosh (c \bar{\zeta}))
$$

maps the coordinates onto the physical domain (denoted by $z=x+\mathrm{i} y$ ) from an intermediate computational domain $(\bar{\zeta}=\bar{\xi}+\mathrm{i} \bar{\eta})$. Furthermore, in the intermediate domain, grid points were clustered near the separation point in the $\bar{\xi}$-direction and near the wall in the $\bar{\eta}$-direction using hyperbolic tangent mappings in order to afford greater resolution in these regions. Consequently, spatial differentiation was performed in an equally spaced rectangular grid. A typical computational grid is shown in figure 8 . Computational, geometrical and other parameters for each run are given in table 1 . A test case with twice as many grid points as case A $(901 \times 201)$ shows that the error associated with the grid resolution in stagnation pressure loss is of the order of $\left|\left(\overline{\Delta p_{t}}\right)_{901 \times 201}-\left(\overline{\Delta p_{t}}\right)_{601 \times 151}\right| /\left(\overline{\Delta p_{t}}\right)_{901 \times 201}=0.8 \%$, where $\overline{\Delta p_{t}}$ denotes the stagnation pressure loss averaged in time and over the cross-section. Since the variation in stagnation pressure loss between different runs is at least an order of magnitude larger in most cases (even when the forcing frequency is varied for the actuation 


\begin{tabular}{cccccccc}
\hline Case & $M_{1}$ & $h_{2} / h_{1}$ & $\theta_{\max }, c$ & $R e$ & $\delta_{b l} / h_{1}$ & $\left(N_{x}, N_{y}\right)$ & $\left(\Delta t a_{2} / h_{2}\right)$ \\
A & 0.6 & $2.0(1.843)$ & $18.43^{\circ}, 0.7576$ & 4000 & 0.10 & 601,151 & $1.0 \times 10^{-3}$ \\
B & 0.2 & $2.0(1.843)$ & $18.43^{\circ}, 0.7576$ & 5000 & 0.10 & 601,151 & $1.0 \times 10^{-3}$ \\
C & 0.4 & $2.0(1.843)$ & $18.43^{\circ}, 0.7576$ & 4000 & 0.10 & 601,151 & $1.0 \times 10^{-3}$ \\
D & 0.8 & $2.0(1.843)$ & $18.43^{\circ}, 0.7576$ & 6000 & 0.10 & 801,201 & $0.8 \times 10^{-3}$ \\
E & 0.6 & $1.4(1.346)$ & $10.78^{\circ}, 0.8440$ & 8000 & 0.07 & 721,181 & $1.0 \times 10^{-3}$ \\
F & 0.6 & $1.6(1.514)$ & $14.03^{\circ}, 0.8110$ & 6670 & 0.08 & 601,151 & $1.0 \times 10^{-3}$ \\
G & 0.6 & $2.6(2.320)$ & $22.31^{\circ}, 0.6992$ & 2900 & 0.13 & 721,181 & $0.727 \times 10^{-3}$ \\
I-O & 0.6 & $2.0(1.843)$ & $18.43^{\circ}, 0.7576$ & 4000 & 0.10 & 601,151 & $1.0 \times 10^{-3}$
\end{tabular}

TABLE 1. Flow conditions and diffuser geometries for the numerical simulation. The values in parentheses in the $h_{2} / h_{1}$ column denote the actual area ratio between the cross-sections at which stagnation pressure profiles were recorded (see figure 8). The Reynolds number is defined as $R e \equiv u_{1} h_{1} / \nu_{1} . \delta_{b l}$ denotes the inlet momentum thickness. $N_{x}$ and $N_{y}$ denote the numbers of grid points in the $x$ - and $y$-directions, respectively, and $\Delta t$ is the time step.

study as discussed in $\S 4.3$ ), the grid resolution is sufficiently high for quantitative comparisons.

Initial velocity fields were calculated based on the potential flow solution, and thermodynamic quantities were found assuming that the flow is homoentropic. The initial velocity and temperature profiles in the boundary layer were specified by solving the Blasiuus boundary-layer equation (cf. §XV of Schlichting 1960) ignoring the curvature of the wall and compressibility. The Prandtl number was set to be $\operatorname{Pr}=0.7$.

We chose the Reynolds number sufficiently high so that vortex shedding occurs (at low Reynolds numbers, the flow becomes a two-dimensional steady jet). Once the Reynolds number exceeds the critical value, the flow pattern becomes fully unsteady and the time-averaged stagnation pressure loss is nearly invariant. For example, a test case with a Reynolds number 1.5 times as high as case A $(R e=6000)$ only decreases the stagnation pressure loss by $\left(\left(\overline{\Delta p_{t}}\right)_{4000}-\left(\overline{\Delta p_{t}}\right)_{6000}\right) /\left(\overline{\Delta p_{t}}\right)_{4000}=8.5 \%$. This is sufficiently small compared with the changes in stagnation pressure loss as the Mach number and the area ratio are varied in this study (refer to §4.2). It should be noted that although the Reynolds number is defined based on $h_{1}$ for consistency, we cannot uniquely define it to describe the whole flow field. For example, the Reynolds number associated with vortex shedding may be $\left(h_{2}-h_{1}\right)$ or $\delta_{b l}$.

No-slip and iso-thermal boundary conditions were imposed on the wall (the upper boundary of the domain), and its temperature was set to be the stagnation temperature of the inflow free stream. A symmetry condition was assumed at $y=0$. Non-reflecting boundary conditions were imposed at both inflow and exit together with a 'sponge' buffer zone (Freund 1997), in which the flow field is forced to relax toward the initial solution. The relaxation coefficient $\sigma$ corresponding to the strength of the sponge is plotted in figure 8 . To implement accurately both non-reflecting and wall-boundary conditions in a non-Cartesian coordinate system, we followed the approach proposed by Visbal \& Gaitonde (1999): A characteristic splitting of the governing equations was performed in the direction normal to the boundary, which is similar to the approach taken by Poinsot \& Lele (1992) for Cartesian coordinates.

\subsection{Periodic mass injection}

To study the effects of periodic mass injection, we artificially forced the right-hand side of continuity, momentum and energy equations near the separation point in the 


$\begin{array}{cccccccc}\text { Case } & \mathrm{I} & \mathrm{J} & \mathrm{K} & \mathrm{L} & \mathrm{M} & \mathrm{N} & \mathrm{O} \\ \omega h_{1} / u_{1} & 0.5 & 0.75 & 1.0 & 1.25 & 1.5 & 2.0 & 2.5\end{array}$

TABLE 2. Frequency of mass flow injection in the numerical simulation. The base flow conditions are the same as case A; namely, $M_{1}=0.6, h_{2} / h_{1}=2.0$, and $R e=4000$ (refer to table 1 ).

$x$-direction:

$$
\begin{gathered}
\frac{\partial \rho}{\partial t}+\frac{\partial\left(\rho u_{j}\right)}{\partial x_{j}}=F\left(x_{1}, x_{2}\right) \rho u_{s}(t), \\
\frac{\partial\left(\rho u_{i}\right)}{\partial t}+\frac{\partial\left(\rho u_{i} u_{j}+p \delta_{i j}+\tau_{i j}\right)}{\partial x_{j}}=F\left(x_{1}, x_{2}\right) \rho u_{s}^{2}(t) \delta_{i 1}, \\
\frac{\partial\left[\rho\left(e+\frac{1}{2} u_{k}^{2}\right)\right]}{\partial t}+\frac{\partial\left[\left\{\rho\left(e+\frac{1}{2} u_{k}^{2}\right)+p\right\} u_{j}+\tau_{j k} u_{k}+q_{j}\right]}{\partial x_{j}} \\
=F\left(x_{1}, x_{2}\right) \rho\left(e+\frac{1}{2} u_{s}^{2}(t)\right) u_{s}(t),
\end{gathered}
$$

where

$$
\begin{aligned}
F(x, y) & =\frac{A_{0}}{2 \pi \sigma_{x} \sigma_{y}} \exp \left[-\frac{\left(x-x_{s}\right)^{2}}{2 \sigma_{x}^{2}}-\frac{\left(y-y_{s}\right)^{2}}{2 \sigma_{y}^{2}}\right], \\
u_{s}(t) & =\frac{1}{2}\left[1+\tanh \left(\frac{t-t_{0}}{\sigma_{t}}\right)\right] \times 0.9 a_{1} \frac{1+\cos (\omega t)}{2},
\end{aligned}
$$

and $\left(x_{s}, y_{s}\right)$ denotes the centre of injection and $a_{1}$ is the speed of sound at the inlet. We chose a non-negative mass flow (i.e. $\sim(1+\cos \omega t)$, cf. Seifert \& Pack 1999) in all cases. The maximum injection velocity was set to be $0.9 a_{1}$, which is a typical experimental condition such that choking is avoided at the injection slot. The forcing terms were distributed in space using a compact but smooth function as shown in (3.5). The injection was gradually activated to minimize spurious transient disturbances. In the computations, $x_{s} / h_{1}=-1.60, y_{s} / h_{1}=1.04, \sigma_{x} / h_{1}=0.08, \sigma_{y} / h_{1}=0.01$, $A_{0}=6.0 \times 10^{-3}, \sigma_{t} a_{1} / h_{1}=0.24$, and $t_{0} a_{1} / h_{1}=0.6$ were selected. Consequently, the momentum coefficient can be calculated as $C_{\mu} \equiv\left(\rho \overline{u_{s}^{2}} h_{s}\right) /\left(\rho u_{1}^{2} h_{1}\right) \approx 0.52 \%, h_{s}$ being the corresponding slot width. Seven different forcing frequencies, $\omega$, were examined (see table 2). The base flow conditions for these runs were the same as case A (unforced).

Although this forcing method can simulate neither the detailed flow near the injector slot nor the internal mechanisms of the actuator, it can capture the key characteristics of periodic mass injection in a computationally efficient fashion. Aside from the frequency dependence, we believe that the most relevant parameters of the actuator are the rate of mass injection (which is characterized by the momentum coefficient, $C_{\mu}$, as used in most previous studies) and the injection velocity, $u_{s}$; accordingly, in simulation we must match the slot width, $h_{s}$, which is typically smaller than $h_{s} / h_{1} \sim 10^{-2}$ in experiments. For example, compared to simply forcing the flow at the boundary by specifying a velocity profile, internal excitation allows more grid points to be clustered in the direction transverse to the jet (see figure 9).

In the following, we evaluate the effects of forcing on the flow to validate this method. Figure 10 shows the dependence of stagnation pressure on the momentum coefficient and the location of the forcing point. The performance is monotonically 


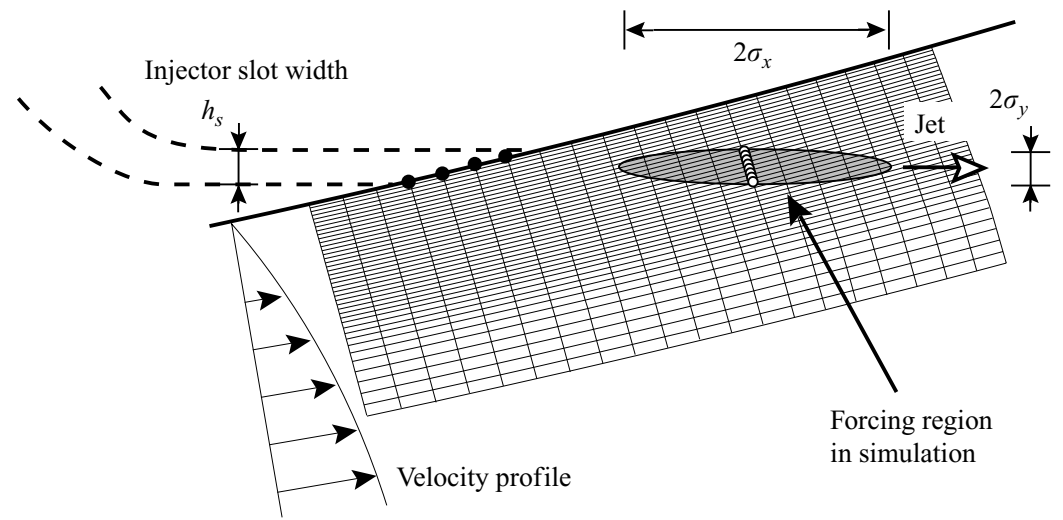

FIGURE 9. Schematic near the forcing region of the simulation. The shaded ellipse denotes the forcing points distributed in the present method, and the points transverse to the jet are denoted by $\circ$. The points which can be used for the forcing on the wall are denoted by
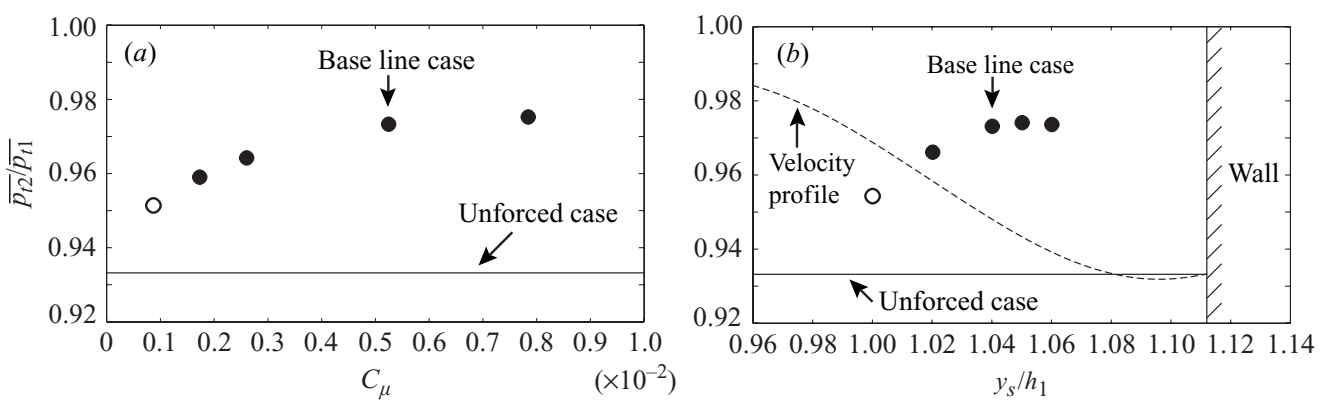

FiguRE 10. Averaged exit stagnation pressure as a function of $(a)$ momentum coefficient and (b) forcing position at $\omega h_{1} / u_{1}=1.25$. Solid symbols, $\bullet$, denote that frequency locking occurs. The velocity profile at the forcing location (arbitrarily scaled) is overlaid in $(b)$.

enhanced as $C_{\mu}$ is increased (via $A_{0}$ in (3.5)) with a magnitude and trend with $C_{\mu}$ similar to previous diffuser experiments (Amitay et al. 2002; Narayanan \& Banaszuk 2003). Furthermore, as the forcing position approaches the wall, the averaged stagnation pressure reaches a plateau (the forcing position cannot approach too closely to the wall because of its finite width). Thus, we demonstrate that this forcing method qualitatively captures the key characteristics of a synthetic-type jet embedded in the upper wall without computing detailed flow configuration.

\section{Results and discussion}

\subsection{Vortex-shedding frequency for unforced cases}

Figure 11 displays vorticity contours from the simulation at several instants in time for case A. Circulation is accumulated downstream of the separation point, in which a large-scale vortex is formed. As this vortex is pinched off and convected downstream, the next vortex is generated. Between these vortices, a counter-rotating (clockwise) vortex is created near the wall. This vortex is convected together with the primary (counter-clockwise) vortex. The flow over the curved part of the wall is almost entirely separated during this process. 

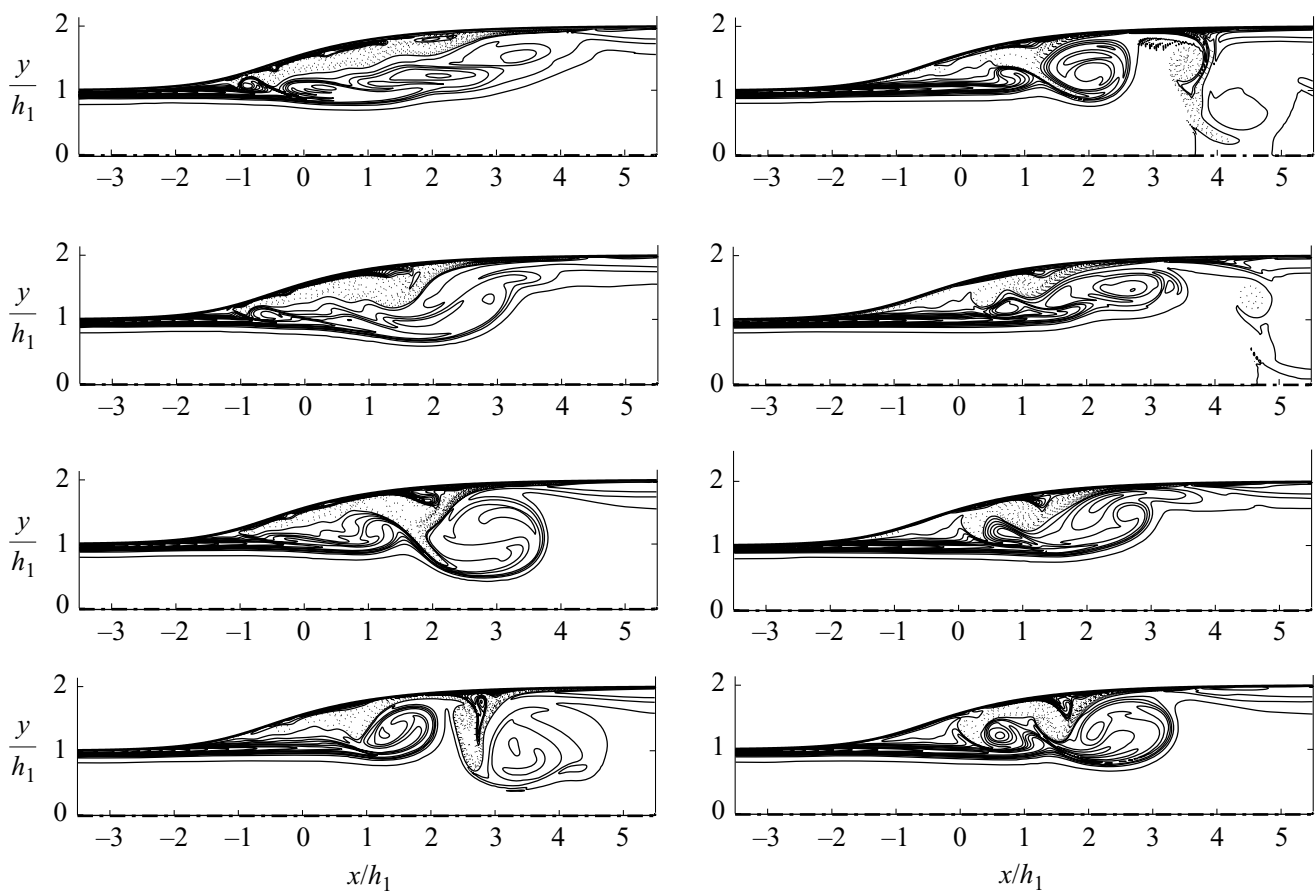

FIGURE 11. Vorticity evolution for case A (unforced). Vorticity contours are shown at: $t u_{1} / h_{1}=57.6,60.0,62.4,64.8,67.2,69.6,72.0$ and 74.4 from the top. Counterclockwise vorticity contours are drawn by solid lines, and clockwise ones by dotted lines. Contour lines: $\omega_{\min }=-33.3, \omega_{\max }=33.3$, and the interval of $\Delta \omega=0.833$.

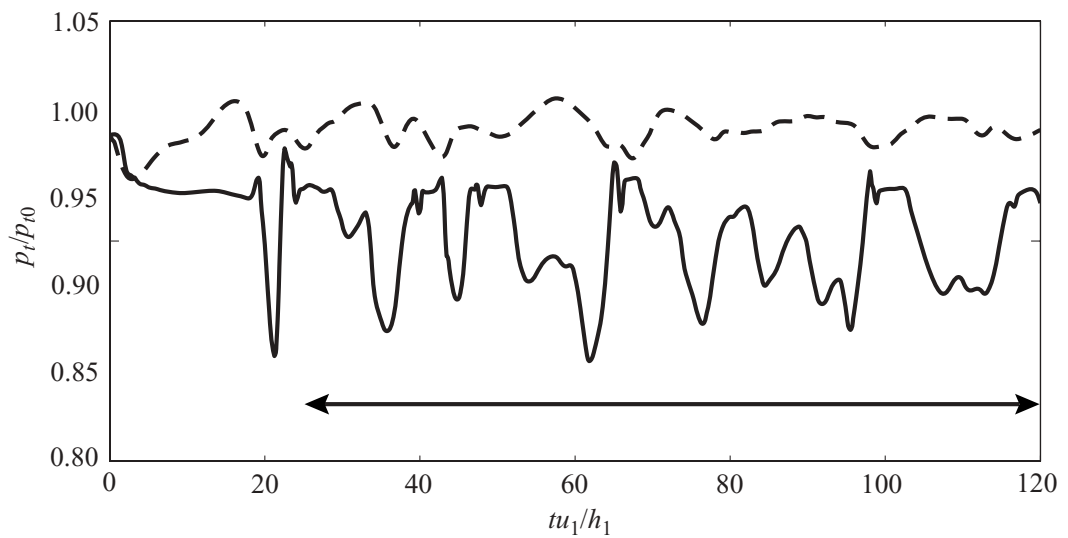

FIGURE 12. Time histories of stagnation pressure in case A. Stagnation pressure was averaged over the cross-section: --- , inlet stagnation pressure $\left(x / h_{1}=-2\right)$; - , exit stagnation pressure $\left(x / h_{1}=3\right) \cdot p_{t 0} \equiv\left(1+(\gamma-1) M_{1}^{2} / 2\right)^{\gamma /(\gamma-1)} p_{1}$.

Figure 12 depicts the time histories of stagnation pressure averaged over the inlet and the exit cross-sections. Although the flow pattern is only quasi-periodic, our model (figure 7) captures features of the exit stagnation pressure profile. Referring to figure 11 , we find that one period of vortex shedding roughly corresponds to an interval between the troughs in the exit stagnation pressure: when the centre of the vortex 

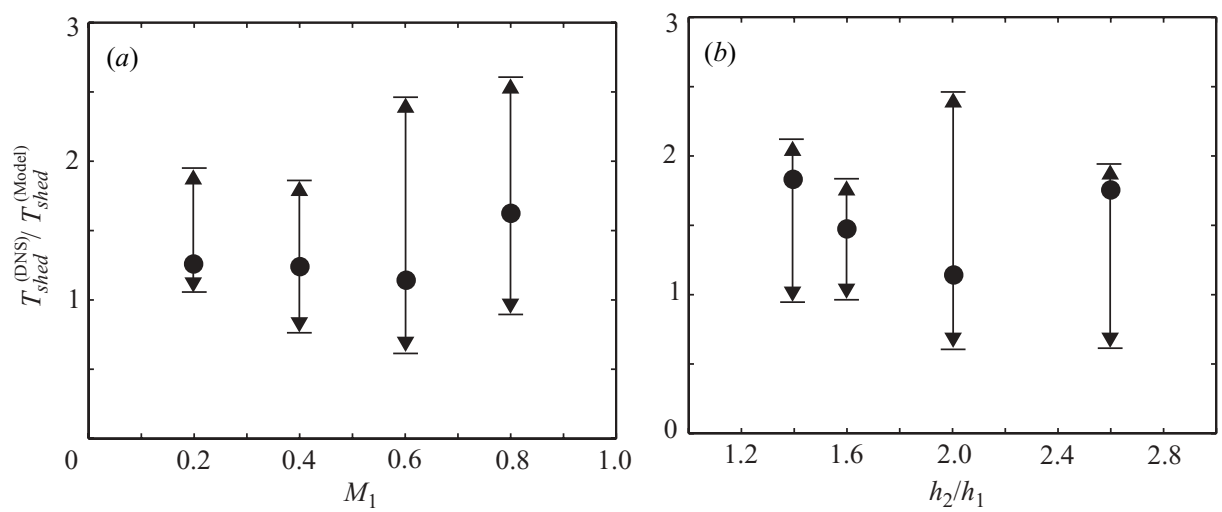

FiguRE 13. Normalized time scale of vortex shedding: $(a)$ Mach number dependence; $(b)$ area ratio dependence. Vortex-shedding time periods from numerical simulations are normalized by the shedding time scale given by (2.8) with $\alpha=1$ and $\lambda=0$. $\bullet$ denotes the peak time period, and the arrows denote the minimum and maximum time periods measured from intervals between troughs. The results of cases B, C, A and D in $(a)$, and cases E, F, A and G in $(b)$, are plotted from left to right.

passes through the exit cross-section, the stagnation pressure drops in a way that is consistent with the analysis of $\S 2.2$.

To validate the inviscid model for the vortex-shedding time period, a total of seven cases was simulated at various Mach numbers and area ratios (cases $A-G$, refer to table 1). The time history of stagnation pressure at the exit was Fourier transformed in each case (a sample period is indicated by arrows in figure 12), and the local peak corresponding to the shedding time period was determined in the frequency domain. Figure 13 plots the ratios of the shedding time period from simulation to the predicted time scale given by (2.8) at different Mach numbers and area ratios. Since the flow is only quasi-periodic as shown in figure 12, the shedding frequency cannot be precisely defined; in fact, it varies over a range as denoted in figure 13. Nonetheless, the peak shedding time periods are within a factor of two of those given by the model. It should be emphasized that without any normalization, the shedding periods differ by nearly a factor of four in the ranges of $M_{1}$ and $h_{2} / h_{1}$ shown in (a) and (b), respectively. Therefore, it is fair to conclude that the shedding time-period scales with the inflow velocity and the function of the area ratio given by the model. We can also deduce that the shedding is less likely to be related to other instabilities, such as local shear layer instability at the separation point or acoustic resonance, which would scale differently with $M_{1}$ and $h_{2} / h_{1}$.

Aside from the cycle-to-cycle variations, the consistent underestimate of the time scale by the model is due in part to inaccurate estimates of the vortex density and size, as well as the induced velocity, $\alpha u_{1}$, to pinch off vortices. For example, $\bar{\rho}$ and $\alpha$ are underestimated by factors of about $3 \%$ and $13 \%$ in case A, respectively. Moreover, the induced velocity of the counter-rotating vortex near the wall probably delays pinching off the primary vortex. As discussed later, although the absorption of circulation from the wall, $\lambda$, is relatively small $(\sim 11 \%)$ in the unforced cases, it can also result in the underestimate of the time scale.

\subsection{Stagnation pressure loss for unforced cases}

Next, the averaged stagnation pressure loss obtained from simulation is compared with the incompressible, inviscid $(\lambda=0)$ and fully separated $\left(\overline{p_{w}}=p_{1}\right)$ model described 



FIGURE 14. Comparison of stagnation pressure loss: $(a)$ Mach number dependence; $(b)$ area ratio dependence. Time-averaged stagnation pressure loss calculated from simulation is compared with the fully separated model in the incompressible limit: pressure loss given by (2.26) $\left(\alpha=1, \beta=1, \lambda=0, \overline{p_{w}}=p_{1}\right.$ and $\kappa=0.33$ are assumed); --- , steady part given by (2.17); $\bullet$, computational results (cases B, C, A and D in (a), and cases E, $\mathrm{F}, \mathrm{A}$ and $\mathrm{G}$ in $(b)$, from left to right). The actual averaged inflow Mach numbers are plotted from the simulations in $(a)$, and the actual area ratios between the inlet and the exit stations specified in table 1 are used for $(b)$.

in $\S 2.2$. The Mach number and area ratio dependence is shown in figure 14. Here, we assume the vortex height to be $h_{3}=\left(\left(h_{1}+h_{2}\right) / 2+h_{1}\right) / 2$ to evaluate $\kappa$. The model captures the trends with the Mach number and the area ratio although the stagnation pressure loss at low Mach numbers is somewhat overestimated (it should be remembered, however, the absolute values of $\overline{p_{t 1}-p_{t 2}}$ between $M_{1}=0.2$ and 0.8 are more than one order of magnitude different). Note that the stagnation pressure loss associated with viscous dissipation is found to be of the order of $10^{-3}$ or less in all cases.

Although we regard the agreement between the model and DNS as satisfactory for our validation purposes, we can observe some discrepancies, which we now discuss. First, the depth of the stagnation pressure troughs in case A (4 to $10 \%$ in figure 12) is somewhat smaller than that of the Oseen vortex model $(\approx 11 \%$ in figure 7$)$. On the other hand, the convective velocity of the vortices in the simulation is nearly half of $u_{2}$ (detailed values of the model coefficients will be given in table 3 and discussed later), while that estimated based on the model is $0.67 u_{2}$ (using (2.23) with $h_{2} / h_{1}=2$ and $\left.h_{3} / h_{1}=5 / 4\right)$. As shown in figure 11 , the convective velocity derived from a singlevortex model is probably reduced by the counter-rotating vortex. Hence, the troughs in the simulation are wider than those predicted by the model, and the unsteady part of the stagnation pressure loss exceeds the prediction. We should also note that the static pressure recovery on the wall provides a positive factor (i.e. $\overline{p_{w}}>p_{1}$ ) in the exit stagnation pressure, which is ignored in the fully separated model in figures 4 and 5. This factor is partially cancelled by the underestimate of the unsteady part mentioned above.

Secondly, from figures 13 and 14 we can postulate that the stagnation pressure loss increases as the flow becomes less periodic. In fact, for the least periodic vortex shedding (when the range of the arrows in figure 13 is the largest), the stagnation pressure loss tends to exceed the prediction by the model. In this case, we observed that vortices tend to be pinched off farther downstream and the flow field in the 


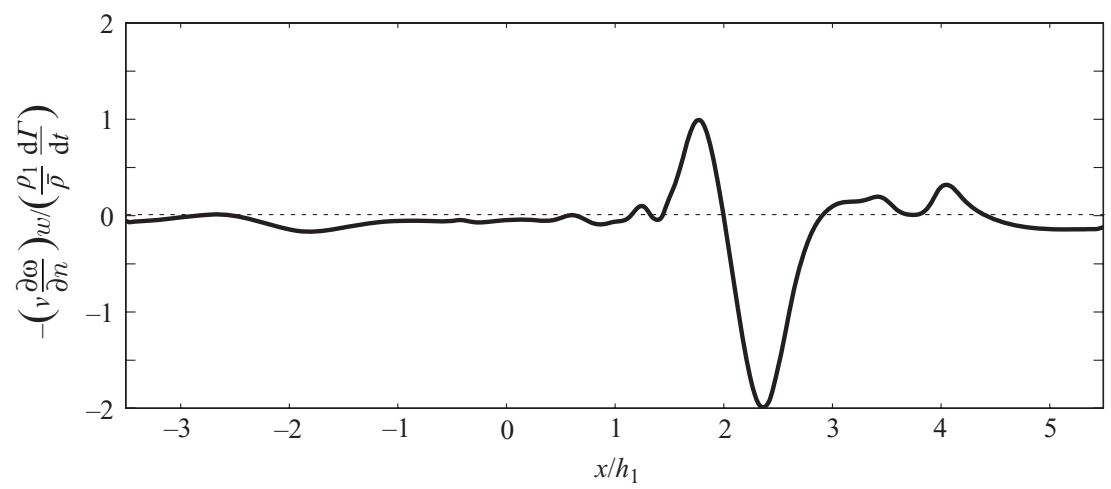

FiguRE 15. Instantaneous vorticity flux from the wall. Vorticity flux from the upper wall is calculated based on (2.27) at $t u_{1} / h_{1}=67.2$ for case A (see figure 11). Production is taken to be positive, and absorption to be negative.

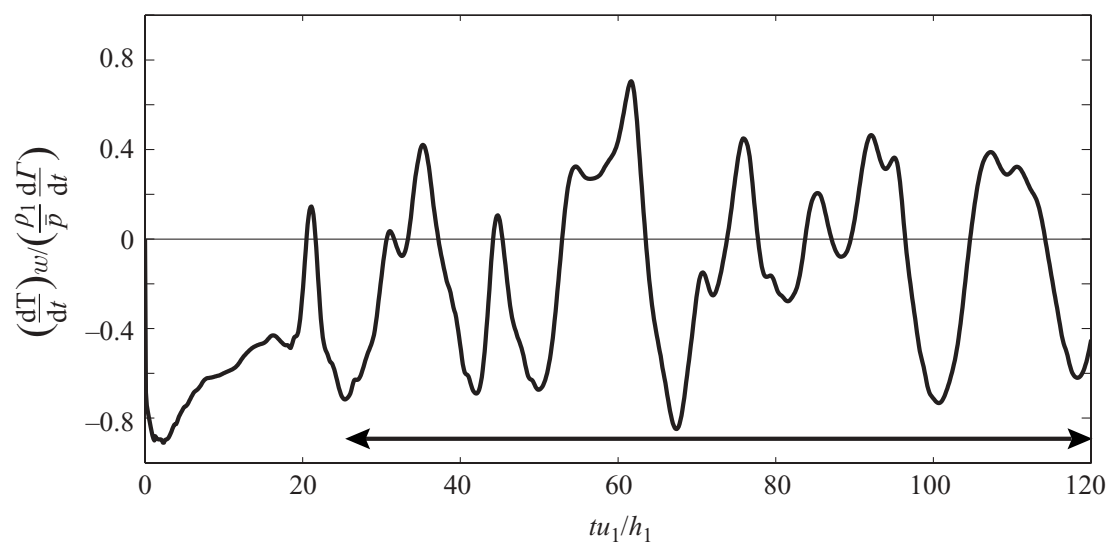

FIGURE 16. Rate of the vorticity flux from the wall. Production is taken to be positive, and absorption to be negative.

separated region includes many small vortices; consequently, the variation in size of pinched-off vortices becomes larger.

Finally, to assess viscous effects at the wall, we plot the computed vorticity flux on the upper wall for case A in figure 15 (at a time corresponding to the top-right in figure 11). It shows that vorticity is absorbed mainly near the leading edge of the vortex. Figure 16 plots the ratio of the net vorticity generation on the wall to the rate of vortex accumulation given by $\left(\left(\rho_{1} / \bar{\rho}\right)(\mathrm{d} \Gamma / \mathrm{d} t)\right)$ in (2.5). From figure 11, we can deduce that some vorticity is absorbed on the wall as the vortex is formed downstream of the separation point. When the vortex convects downstream, the pressure on the wall $\left(p_{2}\right)_{\text {wall }}$ decreases; hence, vorticity is produced at the wall as derived from (2.28). The net rate of absorption in the time interval denoted in figure 16 is about $11 \%$; thus, the net viscous effect is relatively small in the unforced case.

\subsection{Periodic mass injection}

Figure 17 shows the evolution of the vorticity contours for a forced case (case L, which corresponds to the optimal forcing frequency as shown later). Compared with 

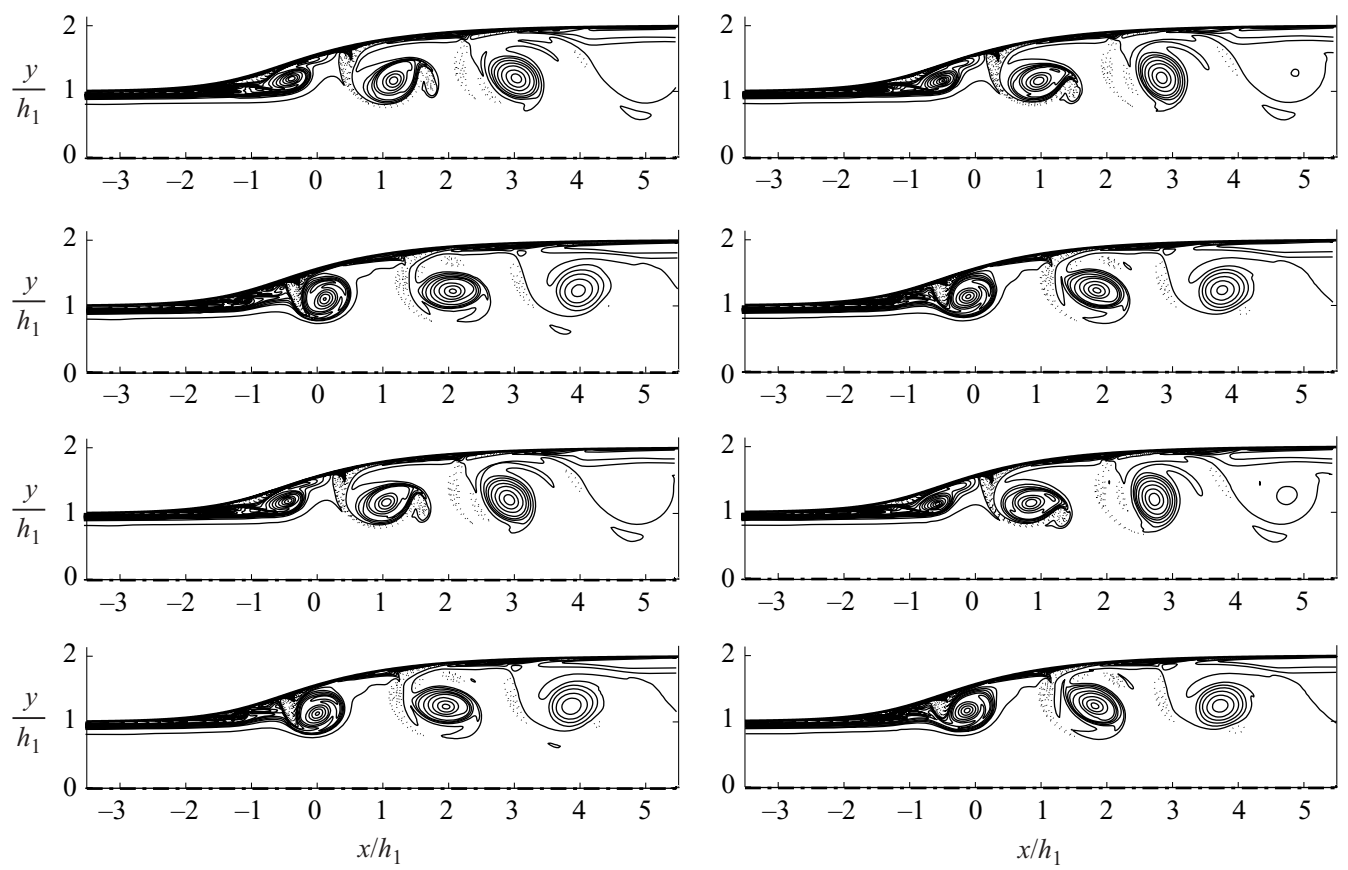

FIGURE 17. Vorticity evolution at the optimal forcing frequency $\left(\omega h_{1} / u_{1}=1.25\right.$, case L). Notation and the time series are the same as figure 11.

(a)

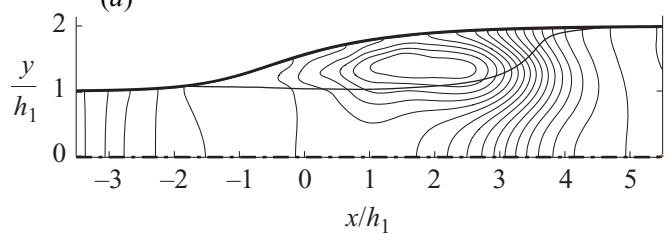

(b)

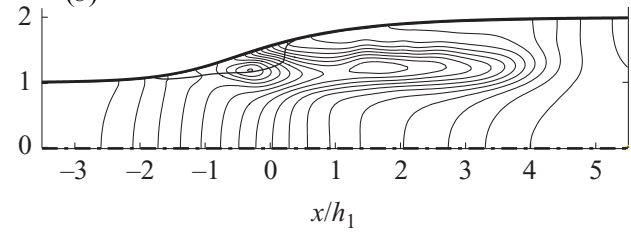

FIGURE 18. Time-averaged pressure contour: $(a)$ the unforced case (case A); $(b)$ the forced case (case L). Contour level: $\bar{p}_{\min } / p_{1}=1.00, \bar{p}_{\max } / p_{1}=1.16$ with the interval of $\Delta \bar{p} / p_{1}=0.01$. Dividing streamlines are overlaid.

the unforced case (figure 11), the size of the vortices is smaller and their convective velocity is higher. The size of the counter-rotating vortices becomes correspondingly smaller. Near the separation point, the next vortex is continuously generated and pinched off before it is developed to the size of the unforced case. Thus, the periodic mass injection tends to reduce $\alpha$ and $\kappa$ in (2.26) and suppresses the unsteady part of the stagnation pressure loss.

Figure 18 compares the time-averaged static pressure field and the dividing streamline for the unforced (case A) and forced (case L) cases. They demonstrate that the reattachment point shifts upstream in the forced case although the apparent separation point does not move. This helps static pressure recovery on the wall and reduces the steady part of the stagnation pressure loss.

Figure 19 depicts the time histories of stagnation pressure at the inlet and exit for case L. Compared with the unforced case (figure 12), the profile is clearly periodic at the forcing frequency (i.e. frequency locking occurs). Moreover, the forced case shows 


\begin{tabular}{ccccccccc}
\hline Case & $\mathrm{A}$ & $\mathrm{I}$ & \multicolumn{1}{c}{$\mathrm{J}$} & $\mathrm{K}$ & $\mathrm{L}$ & $\mathrm{M}$ & $\mathrm{N}$ & $\mathrm{O}$ \\
$\omega h_{1} / u_{1}$ & Unforced & 0.5 & 0.75 & 1.0 & 1.25 & 1.5 & 2.0 & 2.5 \\
$\overline{p_{t 2}} / \overline{p_{t 1}}$ & 0.933 & 0.955 & 0.970 & 0.973 & 0.973 & 0.968 & 0.960 & 0.955 \\
$\overline{p_{w}} / p_{1}$ & 1.049 & 1.055 & 1.065 & 1.070 & 1.074 & 1.075 & 1.071 & 1.065 \\
$\lambda$ & 0.113 & 0.402 & 0.588 & 0.603 & 0.604 & 0.536 & 0.439 & 0.388 \\
$\alpha$ & 1.154 & 0.920 & 0.690 & 0.547 & 0.452 & 0.378 & 0.519 & 0.664 \\
$\beta$ & 0.837 & 0.886 & 1.045 & 0.986 & 0.936 & 1.025 & 0.920 & 1.000 \\
$1-\alpha \kappa$ & 0.531 & 0.578 & 0.678 & 0.771 & 0.782 & 0.746 & 0.663 & 0.557 \\
$h_{3} / h_{1}$ & 1.325 & 1.180 & 1.097 & 1.163 & 1.237 & 1.271 & 1.301 & 1.252
\end{tabular}

TABLE 3. Frequency dependence of each parameter measured from simulation. $\overline{p_{w}}$ and $\lambda$ were directly computed on the upper wall (using (2.27) for $\lambda$ ). $\alpha, \beta$, and $(1-\alpha \kappa)$ were calculated from three samples of vortices passing through the exit cross-section $\left(x_{1} / h_{1}=3\right)$ for each case. A single vortex was defined as a simply supported region of the local vorticity up to $2 \%$ of the peak vorticity.

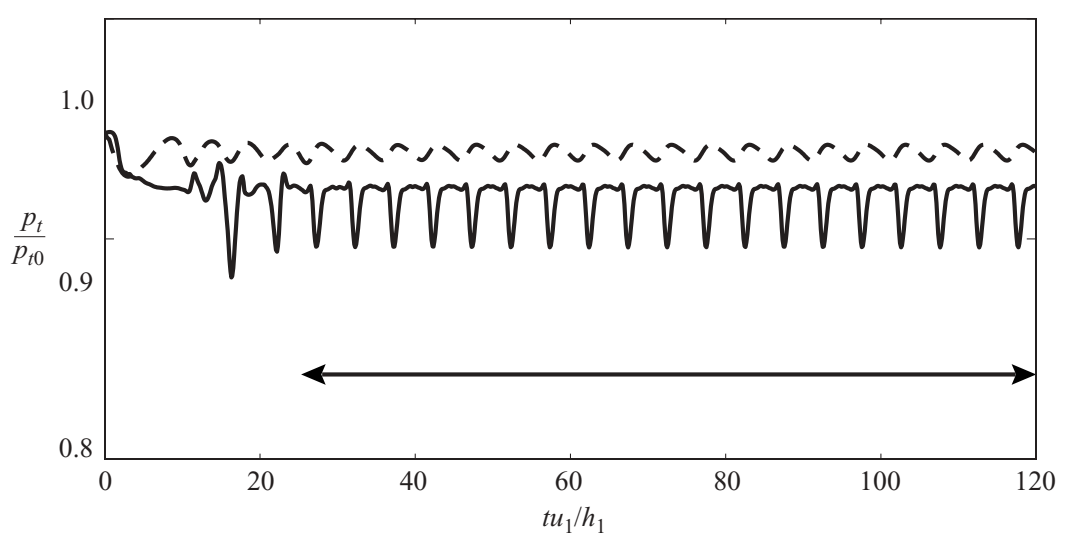

FiguRE 19. Time histories of stagnation pressure at the optimal forcing frequency $\left(\omega h_{1} /\right.$ $u_{1}=1.25$, case $\mathrm{L}$ ). The arrows indicate the period in which the stagnation pressure was evaluated in figure 20. Notation is the same as figure 12 .

sharper stagnation pressure drops and a higher offset; namely, the convective velocity is increased and static pressure on the wall is partially restored.

Next, we study the frequency dependence of periodic mass injection. Figure $20 \mathrm{com}-$ pares the averaged stagnation pressure between the unforced and forced cases over a range of frequencies. Periodic mass injection provides better stagnation pressure at the exit to an appreciable degree $(2 \sim 4 \%)$ in all cases. Note that the direct increase of stagnation pressure owing to mass injection is insignificant $\left(\rho \overline{u_{s}^{2}} h_{s} /\left(2 p_{t 0} h_{1}\right) \approx 2 \times 10^{-3}\right)$. Frequency locking occurs over a range from the natural shedding frequency to roughly twice that frequency and results in substantial improvement in stagnation pressure. At higher frequencies, although vortices continue to be pinched off at the forcing frequency, subsquent vortex pairing apparently causes the stagnation pressure loss to increase (see figure 21 and also Narayanan \& Banaszuk 2003).

The optimal frequency is nearly twice the natural shedding frequency in this flow configuration. In fact, the order of this frequency agrees with the non-dimensional frequency of $F^{+} \equiv f L / u_{\infty} \sim 1$ ( $L$ is a characteristic length and $u_{\infty}$ the free-stream velocity, which we take here as $\left(h_{2}-h_{1}\right)$ and $u_{1}$, respectively). This frequency 


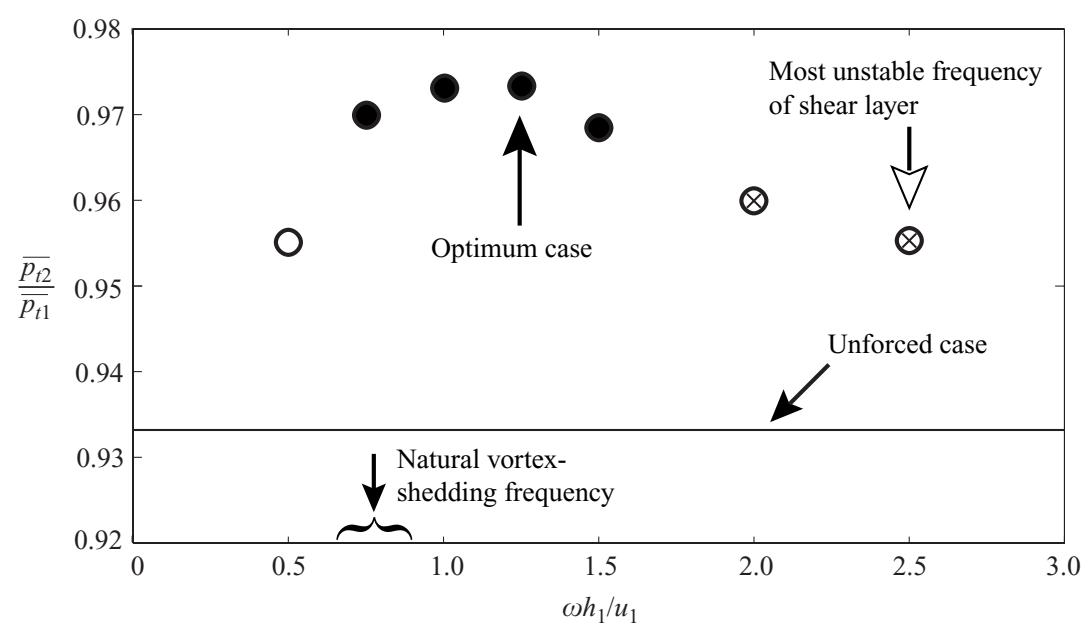

FIgURE 20. Comparison of the exit stagnation pressure at different forcing frequencies. The ratios of the averaged stagnation pressures at the exit $\left(x / h_{1}=3\right)$ to the inlet $\left(x / h_{1}=-2\right)$ are plotted (cases $\mathrm{I}-\mathrm{O}$ from left to right). - indicates that frequency locking occurs, and $\otimes$ indicates that vortex pairing occurs.

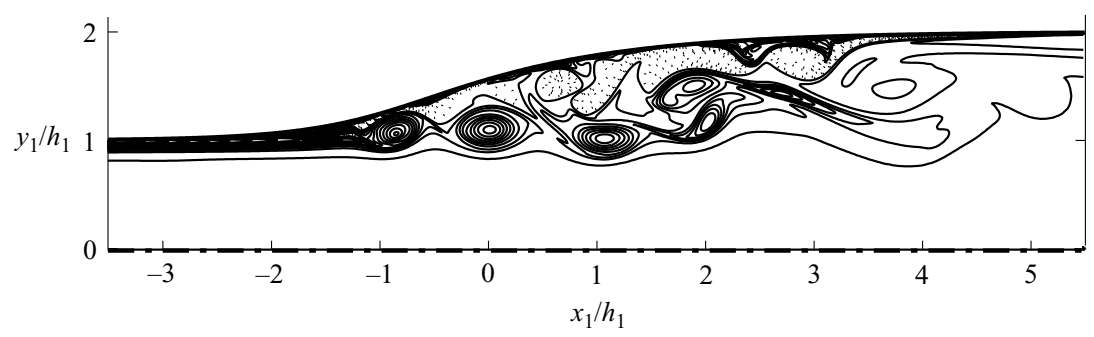

FIGURE 21. A snapshot of vortex pairing. Vorticity contours are drawn (case O). Notation is the same as figure 11.

scale is used in various applications (e.g. Seifert et al. 1996). Even when frequency locking occurs, the highest frequency injection does not necessarily lead to the best performance.

Table 3 shows parameters associated with the model as computed from the simulation results, and figure 22 plots four of these parameters. The correlation between $\overline{p_{t 2}} / \overline{p_{t 1}}$ and $\lambda$ demonstrates that the absorption of circulation helps improve stagnation pressure loss. Even if no frequency locking occurs (case I), the rate of absorption in the forced case is substantially higher than that in the unforced case; similarly, the exit stagnation pressure is also higher in the forced cases. Recall that the mass injection in this study always has a non-negative momentum flux. In the optimal case (case L), the rate of absorption is as much as $60 \%$. As seen in figures 15 , a small vortex generated near the separation point locally creates an adverse pressure gradient and helps absorb vorticity from the wall, as deduced from (2.27).

Attention should be paid to the rate of absorption, $\lambda$, and the convective velocity, $(1-\alpha \kappa)$, which are lower in case M than case L. When a vortex is pinched off into a smaller size by forcing, its centre stays closer to the wall. In fact, $h_{3} / h_{1}$ measured in the simulation follows this trend. Hence, the convective velocity is reduced from (2.23), and the pressure deficit on the wall at the exit is enhanced, i.e. the rate of 

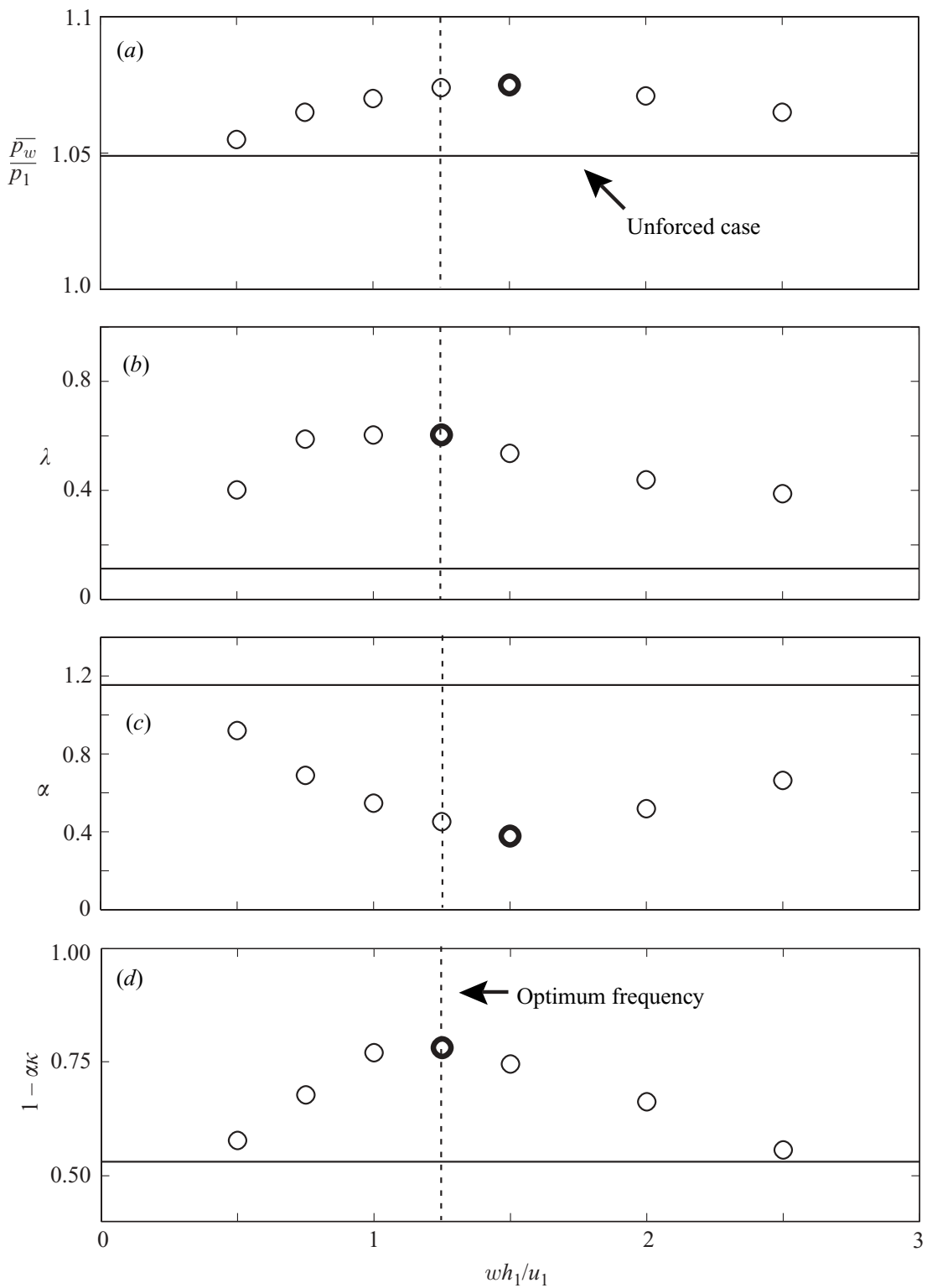

FIGURE 22. Variation of the parameters computed from simulation. The thicker circles denote the optimal value for each quantity.

absorption is decreased from (2.28). This effect seems to provide an upper limit on the reduction of stagnation pressure loss with decreasing $\alpha$. In the unforced case, vortices tend to be pinched off further downstream, and, in turn, the vortex centre is again close to the wall although the size of the vortex is rather large.

If we compare $\alpha$ for different cases in table 3, we find that periodic forcing is effective when the circulation of each vortex is between about a third to a half of the circulation in the unforced case. This agrees with the finding that periodic forcing is most effective when the vortex size is reduced to as little as a third of the separated region in the natural case (Seifert et al. 1996; Seifert \& Pack 1999). However, counter 


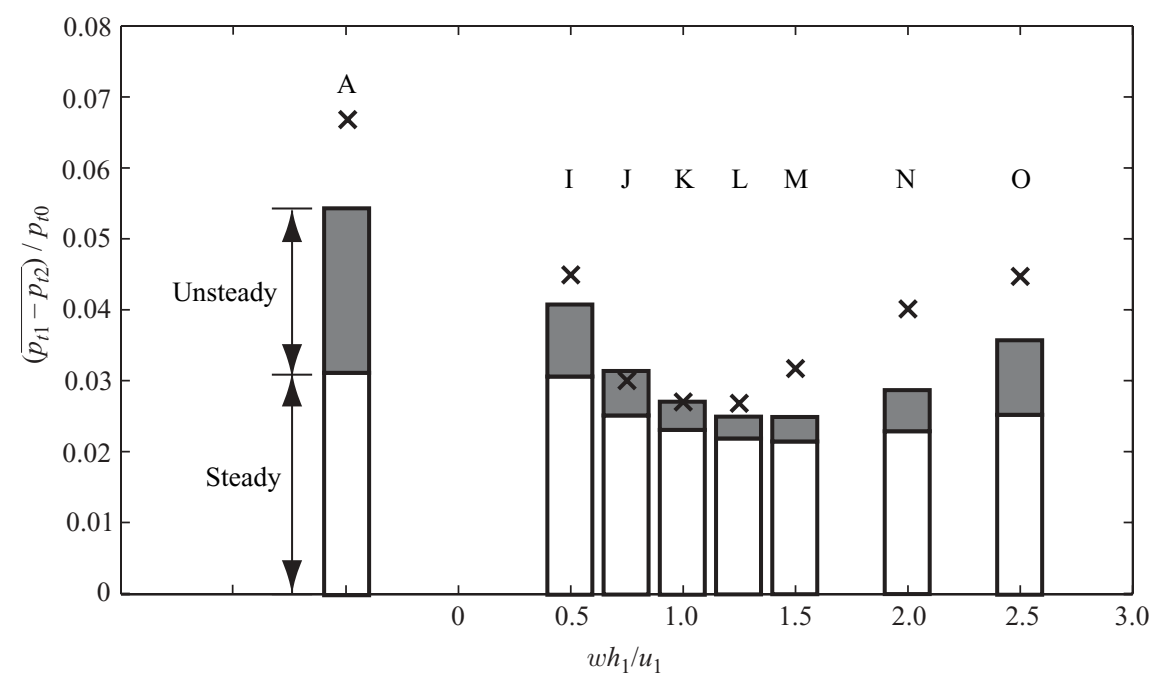

FIGURE 23. Estimated stagnation pressure loss from different components. Parameters in table 3 are substituted into (2.26). The actual total stagnation pressure directly computed from simulation is denoted by $\times$.

to the interpretation given in previous studies (e.g. Asai \& Kaneko 2000), the forcing mechanism is not directly related to instabilities of the shear layer near the separation point. As shown in figure 20, the most unstable frequency of the shear layer estimated from linear stability analysis with inviscid and parallel mean flow assumptions (the mean velocity profile was taken from $x / h_{1}=-0.5$ in case A) is found to be several times higher than the optimal frequency. In experiments, the most unstable frequency should be even higher as the boundary layer becomes thinner (for example, the momentum thickness in an experiment by Narayanan \& Banaszuk 2003 is one order of magnitude thinner). The theoretical discussion in $\S 2$ also implies that the local shear-layer instability does not explicitly govern the forcing mechanism.

Finally, figure 23 depicts the estimated stagnation pressure loss from each component in the model. It indicates that the unsteady part of the stagnation pressure loss is most substantially reduced near the optimal frequency. As the forcing frequency is further increased (at $\omega h_{1} / u_{1}=2.0$ in case $\mathrm{N}$ ), $\alpha$ is nearly doubled because of vortex pairing. Likewise, at $\omega h_{1} / u_{1}=2.5$ in case $\mathrm{O}, \alpha$ is tripled or more because of multiple vortex pairings. Static pressure $\overline{p_{w}} / p_{1}$ is restored for smaller-circulation vortices, but this contribution is weaker than the unsteady part. The underestimates of the net stagnation pressure loss are presumably caused by inaccurate measurement of $\alpha$ and $(1-\alpha \kappa)$, particularly when vortex parings occur (cases $\mathrm{N}$ and $\mathrm{O}$ ) or the flow pattern becomes fully unsteady (cases A and I). In addition, the effects of counterrotating vortices, which are neglected in this chart, result in stagnation pressure losses (stagnation pressure drops owing a vortex regardless of its sign as seen from (2.22)). As mentioned in $\S 2.3$, a negative vorticity flux from the wall generates these vortices in an adverse pressure gradient. Therefore, in addition to increasing the stagnation pressure loss, the counter-rotating vortices prevent absorption of the primary vortex at the wall. 


\section{Conclusions}

We have developed a model that attempts to characterize two aspects of twodimensional vortex shedding in a diffuser. First, the model provides a scaling for the vortex-shedding frequency by accounting for the net circulation accumulated in the separated region. Secondly, in the incompressible limit, the model estimates the stagnation pressure loss, which consists of steady and unsteady parts. Numerical simulations confirm the dependence of these two quantities on the Mach number and the area ratio. The peak shedding frequencies in DNS range from about $50 \%$ to $100 \%$ of the predicted frequencies. Furthermore, the stagnation pressure loss is predicted to within $30 \%$ in most cases, although the unsteady part of the loss tends to be underestimated.

To suppress the stagnation pressure loss, periodic mass injection near the separation point is introduced. Stagnation pressure loss is nearly halved relative to the unforced case when frequency locking is induced. The range of frequency locking is approximately from the natural vortex-shedding frequency to about twice that frequency. As frequency is increased beyond the optimal value, a more subtle degradation in performance occurs owing to the decreases of absorption of circulation at the wall and the convective velocity of the vortices. As the frequency is further increased, vortex pairing occurs and stagnation pressure loss again increases significantly. These results are consistent with the experiments using periodic mass injection (e.g. synthetic jet).

We apply the incompressible model to the study on stagnation pressure loss in the forced cases; in fact, the model represents flow patterns at a fixed frequency even better. The analyses imply that the key to reduce the stagnation pressure loss is to: (i) absorb more circulation from the wall (increase $\lambda$ ); (ii) reduce circulation per vortex (decrease $\alpha$ ); (iii) increase the convective velocity of vortices (decrease $\kappa$ ); (iv) increase the static pressure recovery on the wall (increase $\overline{p_{w}}$ ). In fact, the computational results demonstrate that periodic injection pinches off the separated boundary layer into small vortices immediately downstream of the separation point. Since the centres of the pinched-off vortices become closer to the centreline, their convective velocities are faster than those in the natural shedding case. These vortices locally create an adverse pressure gradient near the separation point and enhance the absorption of circulation from the wall.

Under the present flow conditions, the optimal frequency is approximately twice the natural shedding frequency; however, this value depends on the flow geometry, in particular, via conditions (i) and (iii) above. Hence, it is difficult to find the optimal conditions for these parameters. On the other hand, we expect that conditions (ii) and (iv) can be more easily satisfied if we reduce the size of vortices by increasing the forcing frequency. Furthermore, although we have only simulated laminar diffuser flows, the boundary layer becomes turbulent under practical conditions and this possibly changes the dependence of some parameters. The model developed in this study, however, assumes it to be independent of the Reynolds number and the boundary-layer thickness, except that the rate of absorption can change. This is consistent with the experimental results that the optimal frequency varies weakly with Reynolds numbers (Seifert \& Pack 1999).

Note that the four parameters discussed above are not independent; in fact, three parameters, $\lambda, \alpha$ and $\overline{p_{w}}$, tend to simultaneously shift so that stagnation pressure loss decreases by increasing the forcing frequency until frequency locking breaks. Although the current model can give a crude estimate of $\alpha$ (and possibly $\kappa$ ), it cannot predict $\lambda$ and $\overline{p_{w}}$, especially for forced cases, as a function of the forcing 
frequency. Moreover, figure 10(a) implies that these two parameters are functions of the momentum coefficient as well. Therefore, it is necessary to obtain the relation between the forcing characteristics (i.e. momentum coefficient and frequency) and the parameters (particularly $\lambda$ and $\overline{p_{w}}$ ) to close the model. To actually estimate these parameters, we may need to analyse vortex dynamics in each specific flow geometry.

It should be emphasized that this study focuses on cases in which the separation point is not significantly altered by the forcing. For some applications, the adverse pressure gradient is relatively gentle so that the mass injection can delay the separation point and thereby provide better static pressure recovery. In fact, classical active flow control techniques, such as steady tangential blowing or boundary-layer suction, try to attach the boundary layer. In those studies, the slope angle of the diffuser $\theta$ is considered to be an important parameter. However, the model developed in this study implies that the area ratio governs the large-scale flow unsteadiness for rapidly expanding diffusers. Moreover, the stagnation pressure loss is appreciably reduced even though the boundary layer remains separated. As mentioned above, by pinching off vortices more frequently, we can suppress a substantial part of the unsteady stagnation pressure loss.

We recognize that the model and the simulation developed here lack several features of real diffuser flows. The most obvious limitation is the two-dimensionality although the results, particularly the forcing guidelines, should be applicable to threedimensional diffusers with spanwise coherent vortex shedding (see the discussion of Kaltenbach et al. 1999). In general, we might expect better performance in threedimensional flows than that in two dimensions when the large-scale two-dimensional flow structures are disrupted in the spanwise direction (e.g. Mittal \& Balachandar 1995). However, separation control by periodic forcing still substantially enhances the performance in three dimensions, as many experiments have demonstrated (Amitay et al. 2002; Narayanan \& Banaszuk 2003). We should also stress that our analysis focuses on the transitory stall regime by assuming symmetry. As the area ratio (or the diffuser slope) increases, the flow pattern shifts to 'two-dimensional stall' in a real planar diffuser (Reneau et al. 1967). This is a regime in which the boundary layer separates only on one side and the flow becomes nearly steady again without control.

The authors would like to thank Dr Douglas G. MacMartin for his helpful insights into many aspects of this work. We also thank Mr Jimmy Fung for his help with code development as well as Drs A. P. Dowling, T. P. Hynes and H. Nagib for fruitful discussions. We gratefully acknowledge our collaboration with Dr James D. Paduano under the support of the DARPA (Defense Advanced Research Projects Agency) program (the contract number F49620-00-C-0035).

\section{REFERENCES}

Amitay, M., Pitt, D. \& Glezer, A. 2002 Separation control in duct flows. AIAA J. 39, 616-620.

AsAi, M. \& KANEKo, M. 2000 Experimental investigation of receptivity of a shear layer separating at a convex corner. Fluid Dyn. Res. 27, 117-128.

BACK, L. \& CUFFEL, R. 1982 Experimental investigation of turbulent wall-jets in the presence of adverse pressure gradients in a rectangular diffuser. Intl J. Heat Mass Transfer 25, 871-887.

Batchelor, G. K. 1973 An Introduction to Fluid Dynamics. Cambridge University Press.

Bogar, T. J., Sajben, M. \& Kroutil, J. C. 1983 Characteristic frequencies of transonic diffuser flow oscillations. AIAA J. 21, 1232-1240.

Chu, B.-T. \& Kovásznay, L. S. G. 1958 Non-linear interactions in a viscous heat-conducting compressible gas. J. Fluid Mech. 3, 494-514. 
Freund, J. B. 1997 Proposed inflow/outflow boundary condition for direct computation of aerodynamic sound. AIAA J. 35, 740-742.

Glezer, A. \& Amitay, M. 2002 Synthetic jets. Annu. Rev. Fluid Mech. 34, 503-529.

Hamstra, J. W., Miller, D. N., Truax, P. P., Anderson, B. A. \& Wendt, B. J. 2000 Active inlet flow control technology demonstration. Aeronaut. J. 104, 473-479.

Kaltenbach, H.-J., Fatica, M., Mittal, R., Lund, T. S. \& Moin, P. 1999 Study of flow in a planar asymmetric diffuser using large-eddy simulation. J. Fluid Mech. 390, 151-185.

Koumoutsakos, P., Leonard, A. \& PÉPIN, F. 1994 Boundary conditions for viscous vortex methods. J. Comput. Phys. 113, 52-61.

Kwong, A. H. M. \& Dowling, A. P. 1994 Unsteady flow in diffusers. Trans. ASME I: J. Fluids Engng 116, 842-847.

LELE, S. K. 1992 Compact finite difference schemes with spectral-like resolution. J. Comput. Phys. $103,16-42$.

MacMartin, D. G., Murray, R. M., Verma, A. \& Paduano, J. D. 2001 Active control of integrated inlet/compression systems: initial results. ASME Fluid Engng. FEDSM 2001-18275.

MitTal, R. \& BalachandaR, S. 1995 Effect of three-dimensionality on the lift and drag of nominally two-dimensional cylinders. Phys. Fluids 7, 1841-1865.

Narayanan, S. \& BanaszuK, A. 2003 Experimental study of a novel active separation control approach. AIAA Paper 2003-0060.

Nicoll, W. B. \& RAMAPRiAn, B. R. 1970 Performance of conical diffusers with annular injection at inlet. Trans. ASME D: J. Basic Engng 92, 827-835.

Nishi, M., Yoshida, K. \& Morimitsu, K. 1998 Control of separation in a conical diffuser by vortex generator jets. JSME: Intl J. B 41, 233-238.

Oseen, C. W. 1912 Über Wirbelbewegung in einer reibenden Flüssigkeit. Ark. f. Mat. Astron. Fys. 7, 14.

Pierrehumbert, R. T. 1980 A family of steady, translating vortex pairs with distributed vorticity. J. Fluid Mech. 99, 129-144.

Poinsot, T. J. \& Lele, S. K. 1992 Boundary conditions for direct simulations of compressible viscous flows. J. Comput. Phys. 101, 104-129.

RAO, D. M. 1971 A method of flow stabilisation with high pressure recovery in short, conical diffusers. J. R. Aeronaut. Soc. TN 75, 336-339.

Reneau, L. R., Johnston, J. P. \& Kline, S. J. 1967 Performance and design of straight, twodimensional diffusers. Trans. ASME D: J. Basic Engng 89, 141-150.

Saffman, P. G. 1992 Vortex Dynamics. Cambridge University Press.

Salmon, J. T., Bogar, T. J. \& Sajben, M. 1983 Laser Doppler velocimeter measurements in unsteady, separated, transonic diffuser flows. AIAA J. 21, 1690-1697.

Schlichting, H. 1960 Boundary Layer Theory, 4th edn (translated by J. Kestin) McGraw-Hill.

Seifert, A., Darabi, A. \& Wygnanski, I. 1996 Delay of airfoil stall by periodic excitation. J. Aircraft 33, 691-698.

SeIfERT, A. \& PACK, L. G. 1999 Oscillatory control of separation at high Reynolds numbers. AIAA J. 37, 1062-1071.

SмItH, C. R. 1978 Transitory stall time-scale for plane-wall air diffuser. Trans. ASME I: J. Fluids Engng 100, 133-135.

Smith, C. R. \& Kline, S. J. 1974 An experimental investigation of the transitory stall regime in two-dimensional diffuser. Trans. ASME D: J. Fluids Engng 96, 11-15.

Tennant, J. S. 1973 A subsonic diffuser with moving walls for boundary-layer control. AIAA J. 11, 240-242.

Visbal, M. R. \& Gaitonde, D. V. 1999 High-order-accurate methods for complex unsteady subsonic flows. AIAA J. 37, 1231-1239.

VINOKUR, M. 1974 Conservation equation of gas-dynamics in curvilinear coordinate systems. J. Comput. Phys. 14, 105-125.

WeLsh, M. C. 1976 Flow control in wide-angled conical diffusers. Trans. ASME D: J. Fluids Engng 98, 728-735. 